ARTICLE

https://doi.org/10.1038/s41467-019-10932-4

\title{
Zinc-binding to the cytoplasmic PAS domain regulates the essential WalK histidine kinase of Staphylococcus aureus
}

Ian R. Monk ${ }^{1,8}$, Nausad Shaikh2,8, Stephanie L. Begg1, Mike Gajdiss ${ }^{3}$, Liam K.R. Sharkey (1) 1, Jean Y.H. Lee (D) 1, Sacha J. Pidot (10 1, Torsten Seemann (1) 1,4, Michael Kuiper ${ }^{5}$, Brit Winnen, Rikki Hvorup², Brett M. Collins ${ }^{2}$, Gabriele Bierbaum $^{3}$, Saumya R. Udagedara ${ }^{6}$, Jacqueline R. Morey (1) ${ }^{7}$, Neha Pulyani ${ }^{6}$, Benjamin P. Howden (1) ${ }^{1}$, Megan J. Maher (10 6, Christopher A. McDevitt (1) ${ }^{1,7,9}$, Glenn F. King ${ }^{2,9}$ \& Timothy P. Stinear (1) 1,9

WalKR (YycFG) is the only essential two-component regulator in the human pathogen Staphylococcus aureus. WalKR regulates peptidoglycan synthesis, but this function alone does not explain its essentiality. Here, to further understand WalKR function, we investigate a suppressor mutant that arose when WalKR activity was impaired; a histidine to tyrosine substitution (H271Y) in the cytoplasmic Per-Arnt-Sim (PASCYT) domain of the histidine kinase WalK. Introducing the WalK ${ }^{\mathrm{H} 271 Y}$ mutation into wild-type $S$. aureus activates the WaIKR regulon. Structural analyses of the WaIK PASCYT domain reveal a metal-binding site, in which a zinc ion $\left(\mathrm{Zn}^{2+}\right)$ is tetrahedrally-coordinated by four amino acids including H271. The WalK ${ }^{\mathrm{H} 271 Y}$ mutation abrogates metal binding, increasing WalK kinase activity and WalR phosphorylation. Thus, $\mathrm{Zn}^{2+}$-binding negatively regulates WalKR. Promoter-reporter experiments using $S$. aureus confirm $\mathrm{Zn}^{2+}$ sensing by this system. Identification of a metal ligand recognized by the WalKR system broadens our understanding of this critical $S$. aureus regulon.

\footnotetext{
${ }^{1}$ Department of Microbiology and Immunology, Doherty Institute for Infection and Immunity, University of Melbourne, Melbourne, VIC 3000, Australia. ${ }^{2}$ Institute for Molecular Bioscience, The University of Queensland, St Lucia, QLD 4067, Australia. ${ }^{3}$ University Clinics of Bonn, Institute of Medical Microbiology, Immunology and Parasitology, 53127 Bonn, Germany. ${ }^{4}$ Melbourne Bioinformatics, University of Melbourne, Melbourne, VIC 3000 , Australia. ${ }^{5}$ CSIRO Data61, Canberra, ACT 2601, Australia. ${ }^{6}$ Department of Biochemistry and Genetics, La Trobe Institute for Molecular Science, La Trobe University, Melbourne, VIC 3086, Australia. 7 Department of Molecular and Biomedical Sciences, School of Biological Sciences, The University of Adelaide, Adelaide, SA 5005, Australia. ${ }^{8}$ These authors contributed equally: lan R. Monk, Nausad Shaikh. ${ }^{9}$ These authors jointly supervised this work: Christopher A. McDevitt, Glenn F. King, Timothy P. Stinear. Correspondence and requests for materials should be addressed to I.R.M. (email: ian.monk@unimelb.edu.au) or to T.P.S. (email: tstinear@unimelb.edu.au)
} 
S taphylococcus aureus is a major human pathogen that causes a wide range of hospital- and community-acquired opportunistic infections ${ }^{1}$. Antibiotic-resistant strains (in particular methicillin-resistant $S$. aureus [MRSA]) are increasing in prevalence in both hospital and community settings. Resistance to last line agents such as vancomycin, linezolid and daptomycin is well described ${ }^{2,3}$, casting doubt on their efficacy for the treatment of serious MRSA infections ${ }^{4,5}$. In the context of limited treatment options, the social and financial burden posed by $S$. aureus-related disease is now globally significant ${ }^{1}$.

WalKR is a highly conserved two-component regulatory system (TCS) with features that are unique among low G+C Grampositive bacteria $^{6}$. Like other TCS, it comprises a multi-domain transmembrane sensor histidine kinase (WalK-HK) (Fig. 1a) and a response regulator (WalR-RR). Notably, in S. aureus and closely related bacteria, the locus is essential, a characteristic that has made it a potential therapeutic target ${ }^{7}$. The WalR/WalK system (also called YycFG and VicRK) was first identified from temperature-sensitive mutants in Bacillus subtilis ${ }^{8}$ and then $S$. aureus $^{9}$, with the system essential in both genera. Essentiality of WalKR has been inferred through both construction of strains containing an inducible WalKR ${ }^{10,11}$, which were unable to grow in the absence of inducer, and the inability to delete the genes in Listeria monocytogenes or Enterococcus faecalis ${ }^{12,13}$. In an impressive recent study, Villanueva et al. ${ }^{14}$ deleted all 15 TCSs from two different $S$. aureus backgrounds and confirmed that WalKR is the only TCS strictly required for growth. Depletion of WalKR in B. subtilis produces a long-chain phenotype with the formation of multiple 'ghost' cells without cytoplasmic contents, which correlates with a reduction in colony-forming units (CFUs) ${ }^{15}$. In $S$. aureus, depletion of WalKR causes the same loss in viability, but without cellular lysis, although aberrant septum formation was reported ${ }^{16}$. These observations suggest different mechanisms for the essentiality of WalKR in rod- versus coccusshaped bacteria. However, this is not a uniform requirement as, despite the presence of the TCS in the streptococci, the WalK orthologue in this species is not essential and downstream regulators are absent, suggesting altered regulation. Further, WalK in streptococci contains only one transmembrane domain, which differentiates it from other cocci 6 .

S. aureus antibiotic resistance and clinical persistence are frequently linked to mutations in regulatory genes, in particular TCSs. Among the S. aureus TCSs, mutations in loci such as vraRS, graRS and walKR are associated with the vancomycin intermediate resistant $S$. aureus (VISA) phenotype ${ }^{17-19}$. Notably, mutations in WalKR are a critical contributor towards this phenotype with numerous clinical VISA strains possessing walKR mutations ${ }^{20}$. Induction of characteristic VISA phenotypes (thickened cell wall, reduced autolytic activity and reduced virulence) can arise from mutations as simple as a singlenucleotide change in either walK or walR w $^{2-23}$.

Despite the central role of the TCS in bacterial viability, the physiological and/or mechanical signal sensed by WalK is unknown. In B. subtilis, where it has been more extensively studied, WalK localises to the division septum and interacts with proteins of the divisome ${ }^{24}$. B. subtilis WalR positively regulates several autolysins involved in peptidoglycan metabolism and represses inhibitors of these enzymes ${ }^{25}$. Thus WalKR has been inferred to link cell division with cell wall homeostasis ${ }^{6,24}$. Nevertheless, its role in $S$. aureus appears to be distinct. Although $S$. aureus WalR does control autolysin expression ${ }^{16,26}$, this function does not explain the essentiality of the system as expression of genes encoding lytic transglycosylases or amidases in a WalKR-depleted strain do not restore cell viability ${ }^{27}$. Further, the membrane-associated regulators $\mathrm{YycHI}$ act as an activator of
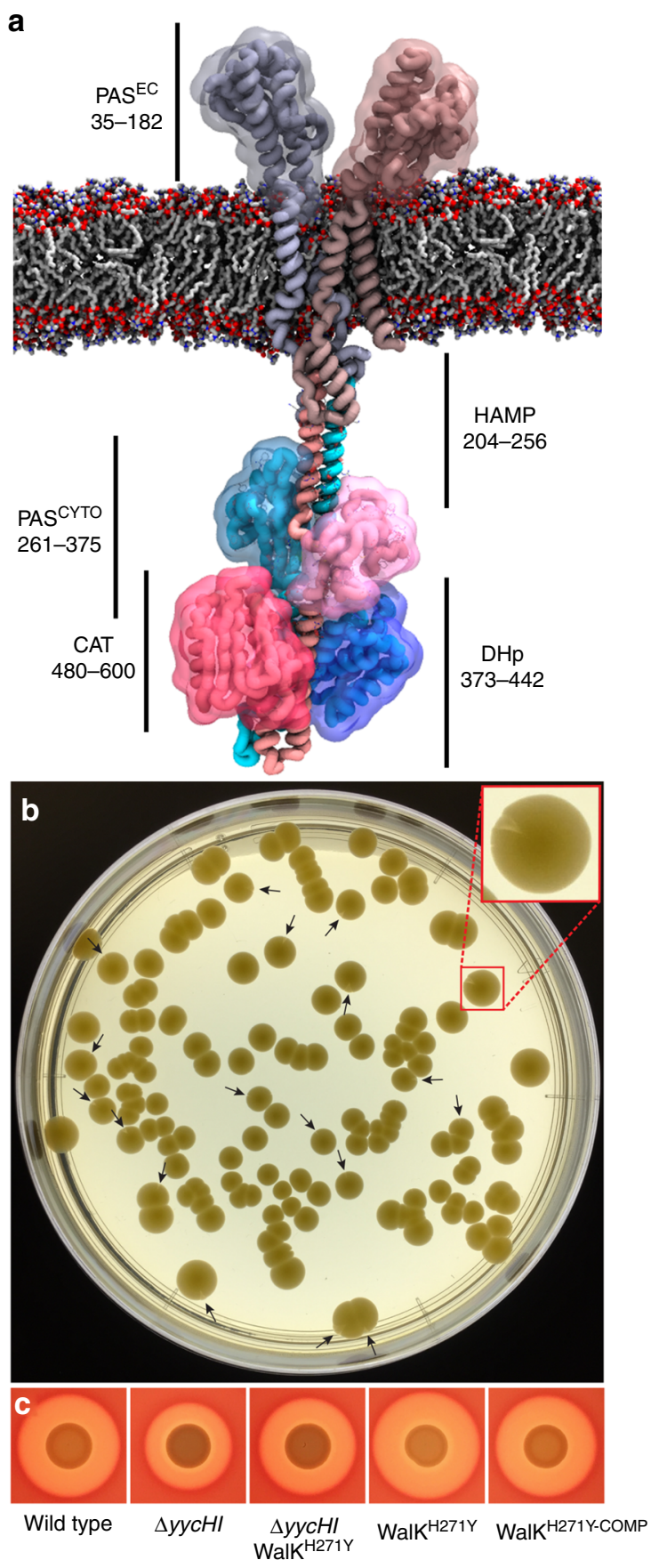

Fig. 1 Colony sectoring in a $\Delta y y c H I$ leads to mutation in WalK. a Molecular model of the essential two-component histidine kinase WalK (dimer 608 residues) from $S$. aureus in a phospholipid bilayer. The amino acid boundaries of the various WalK domains (PASEC: extracellular PAS, HAMP: present in Histidine kinases, Adenylate cyclases, Methyl accepting proteins and Phosphatases, PASCYT: cytoplasmic PAS, DHp: dimerisation and histidine phosphorylation, CAT: catalytic/ATP binding) are highlighted. b Plating of NRS384 $\Delta y y c H$ I onto Brain Heart Infusion agar after $48 \mathrm{~h}$ at $37^{\circ} \mathrm{C}$ promotes colony sectoring (arrow heads). Red box inset shows enlarged view of one sectored colony. c The WalK ${ }^{\mathrm{H} 271 Y}$ mutation identified from whole-genome sequencing of a single sectored colony was introduced by allelic exchange into the NRS384 background, with the mutation increasing haemolysis on sheep blood agar. Also shown are the wildtype (NRS384), $\Delta y y c H I, \Delta y y c H I W a I K^{H 271 Y}$ and the WalK ${ }^{H 271 Y-C O M P}$, for reference 
WalK function in $S$. aureus $^{28}$, which contrasts with $B$. subtilis where they serve to repress the system ${ }^{29}$.

Sequence variation provides one basis for the apparent differences in WalKR function between S. aureus and B. subtilis. The WalK alleles share only $45 \%$ amino acid identity, with the majority of the variation concentrated in the extracellular region and a cytoplasmic Per-Arnt Sim (PAS) domain (Fig. 1a). Although these regions have low sequence conservation, PAS domains are known to adopt a conserved tertiary fold, where they facilitate sensory perception via ligand interaction, enabling signal transduction. In HKs that contain PAS domains, known ligands include haeme, flavin mononucleotide and di/tricarboxylic acids ${ }^{30}$. In B. subtilis, the cytoplasmic PAS domain is essential for WalK localisation to the division septum ${ }^{24}$. Recently, S. aureus WalK was also shown via a green fluorescent protein fusion to be preferentially located to the division septum, but the role of the cytoplasmic PAS domain in septum targeting was not examined $^{31}$.

Here we screen $S$. aureus $y y c H I$ deletion mutants and identify a WalK-suppressor mutation (H271Y) located in the cytoplasmic PAS domain. Structural and functional analyses of WalK reveal that this residue is a critical component of a cytoplasmic metalbinding site that directly influences HK activity. This metalloregulatory site binds zinc $\left(\mathrm{Zn}^{2+}\right)$ in vitro, and this is abrogated by the H271Y mutation resulting in increased WalK autophosphorylation. We could verify the potential metalloregulation of WalK through the construction of a metal-binding site S. aureus mutant at the native locus with subsequent activation of WalKRassociated phenotypes and increases in WalR phosphorylation in vivo. Further, exogenous supplementation with zinc directly influenced the expression WalR-controlled genes. Molecular modelling of WalK in $\mathrm{Zn}^{2+}$-free and $\mathrm{Zn}^{2+}$-bound states suggests that metal occupancy influences conformational changes associated with the cytoplasmic domains, thus providing a plausible mechanism of activity modulation. Thus we show that metal binding to the cytoplasmic PAS domain can influence the activity of a HK.

\section{Results}

Mapping of a suppressor mutation to walK by genome sequencing. $\mathrm{YycH}$ and $\mathrm{YycI}$ are membrane-associated accessory proteins that have been shown to directly interact with WalK and positively regulate WalKR function ${ }^{28}$. We constructed an $S$. aureus $y y c H I$ deletion mutant $(\Delta y y c H I)$ in USA300 strain NRS384 and observed colony sectoring after 2 days growth at $37^{\circ} \mathrm{C}$ on Brain Heart Infusion (BHI) agar (Fig. 1b). This observation suggested that the $\triangle y y c H I$ mutation yielded genetic instability under the growth conditions, due to altered regulation of the essential TCS, which permitted the development of suppressor (compensatory) mutations. We purified the two colony morphotypes and performed whole-genome sequencing. Aligning the sequence reads to the closed NRS384 genome revealed only a single point mutation in addition to the engineered $y y c H I$ deletion. The mutation occurred in the cytoplasmic PAS domain of WalK, wherein histidine 271 was replaced by tyrosine (WalK ${ }^{\mathrm{H} 271 Y}$ ). Despite the different chemical profiles of the two side chains, i.e. positively charged versus slightly polar, the two residues have similar steric bulk.

Generation of a walK $^{\mathrm{H} 271 \mathrm{Y}}$ site-directed mutant. We next sought to investigate the impact of the WalK ${ }^{\mathrm{H} 271 \mathrm{Y}}$ substitution by introducing the mutation into wild-type NRS384 by allelic exchange. The resulting unmarked mutant was analysed by genome sequencing to exclude the occurrence of secondary site mutations, as was previously observed to occur in backgrounds involving mutagenesis of WalK ${ }^{22,32}$. Here only the nucleotides targeted by the allelic exchange of walK differed between wildtype walK and the walK $\mathrm{K}^{\mathrm{H} 271 \mathrm{Y}}$ mutant. The allelic exchange procedure was then repeated to convert the wal $\mathrm{K}^{\mathrm{H} 271 \mathrm{Y}}$ allele back to wild-type with the introduction of a silent PstI restriction site to mark the revertant (walk $\left.K^{\mathrm{H} 271 Y-C O M P}\right)$. By comparison with the wild-type, the WalK $\mathrm{K}^{\mathrm{H} 271 \mathrm{Y}}$ mutant formed smaller colonies on sheep blood agar with reduced pigmentation but produced a slightly larger zone of haemolysis (Fig. 1c). Deletion of $y y c H I$ resulted in reduced haemolysis. However, haemolysis was elevated upon the introduction of the $\mathrm{WalK}^{\mathrm{H} 271 \mathrm{Y}}$ allele into the $\Delta y y c H I$ background (Fig. 1c). Intriguingly, following growth in Tryptone soy broth (TSB) at $37^{\circ} \mathrm{C}$, the WalK $\mathrm{K}^{\mathrm{H}} 71 \mathrm{Y}$ strain did not exhibit a lag phase upon inoculation into fresh medium (Fig. 2a). Nonetheless, at $2 \mathrm{~h}$ post inoculation the growth rate was significantly reduced in comparison with the wild-type and complemented strain. The maximal doubling rate was $33 \mathrm{~min}$ for the WalK ${ }^{\mathrm{H} 271 \mathrm{Y}}$ mutant versus 23 min for the wild-type and complemented strains. Further, the WalK $\mathrm{H}^{\mathrm{H} 271 \mathrm{Y}}$ mutant strain had a reduced final optical density at $600 \mathrm{~nm}\left(\mathrm{OD}_{600}\right)$ compared to the wild type, although this equated to identical CFU counts (Fig. 2a).

The exoproteome and Atl activity is altered in $\mathrm{WalK}^{\mathrm{H} 271 \mathrm{Y}}$ mutant. Atl is a major peptidoglycan hydrolase produced by $S$. aureus that is positively controlled by WalKR ${ }^{16,26}$. It is involved in daughter cell separation and also plays roles in primary attachment during biofilm formation and in the secretion of moonlighting proteins $\mathrm{s}^{33}$. Here, we analysed the secretion of proteins during exponential and stationary phase growth from the wild-type and the mutant $\mathrm{WalK}^{\mathrm{H} 271 \mathrm{Y}}$, WalK ${ }^{\mathrm{H} 271 \mathrm{Y}-\mathrm{COMP}}$ and $\Delta$ atl strains. We observed an increase in protein abundance in the WalK ${ }^{\mathrm{H} 271 \mathrm{Y}}$ mutant in both exponential and stationary phases, by comparison to the wild-type levels observed in the Wal$\mathrm{K}^{\mathrm{H} 271 Y \text {-COMP }}$ strain. In contrast, secreted protein levels were reduced in the $\Delta a t l$ strain compared to the wild type (Fig. 2b). We then directly assessed Atl activity in the exoproteome by zymogram analysis, using Micrococcus luteus cells as a substrate. The $\Delta a t l$ strain displayed no visible lytic activity in contrast to the other strains, indicating that the majority of lysis is attributable to $\mathrm{Att}^{34}$ (Fig. 2c). In stationary phase, there was an apparent increase in the amidase $(63 \mathrm{kDa})$ and glucosaminidase $(53 \mathrm{kDa})$ activity in samples from the WalK ${ }^{\mathrm{H} 271 Y}$ mutant. Using a Patl-YFP reporter plasmid, we observed a significant 1.7 -fold increase in at expression in the walk $\mathrm{H}^{\mathrm{H} 21 \mathrm{Y}}$ mutant over the wild type $(p<0.05$, Student's $t$ test, Supplementary Fig. 1a). These data show that the WalK ${ }^{\mathrm{H} 271 Y}$ mutation results in increased expression and production of the WalR-regulated Atl.

Increased lysostaphin and vancomycin sensitivity of Wal$\mathrm{K}^{\mathrm{H} 271 \mathrm{Y}}$ mutant. WalKR regulates autolysis and directly influences sensitivity to vancomycin and lysostaphin ${ }^{16,22,23,32}$. Here we used lysostaphin and vancomycin sensitivity as indirect measures of WalKR activity. After a 90-min exposure to $0.2 \mu \mathrm{g} \mathrm{ml}^{-1}$ of lysostaphin, NRS384 exhibited a $0.5-\log _{10}$ reduction in cell viability, while the WalK ${ }^{\mathrm{H} 271 Y}$ mutant showed a further $0.5-\log _{10}$ increase in sensitivity. Complementation returned lysostaphin sensitivity to wild-type levels (Fig. 2d). In contrast, the $\Delta y y c H I$ mutant displayed increased lysostaphin resistance compared to the wild type, consistent with YycHI positively regulating WalKR. Therefore, the knockout of $y y c H I$ leads to reduced WalKR-controlled autolytic activity (Fig. $2 \mathrm{~d})^{28}$. The compensatory WalK ${ }^{\mathrm{H} 271 \mathrm{Y}}$ mutation in the $\Delta y y c H I$ background did not fully restore lysostaphin sensitivity to wild-type levels. However, increased sensitivity to lysostaphin was observed at a higher concentration $\left(1 \mu \mathrm{g} \mathrm{ml}^{-1}\right)$ with the $\Delta y y c H I-$ WalK ${ }^{\mathrm{H} 271 \mathrm{Y}}$ strain $2-\log _{10}$ less viable than the $\Delta y y c H I$ strain 
a

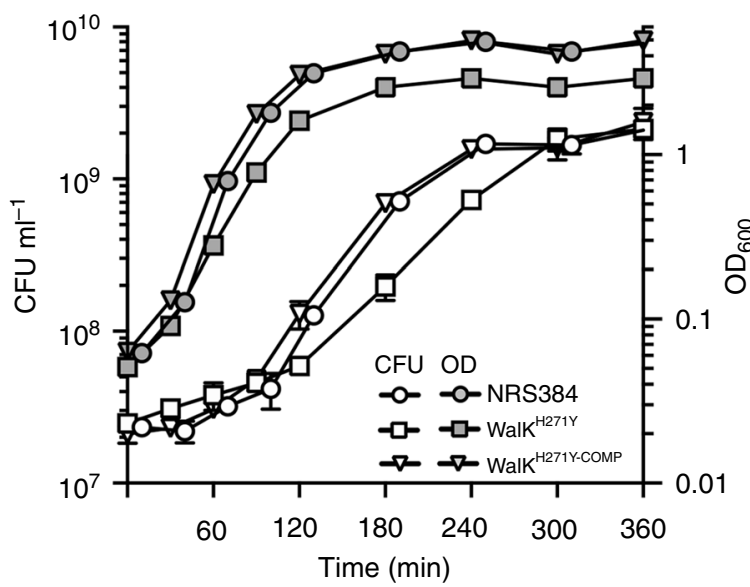

b

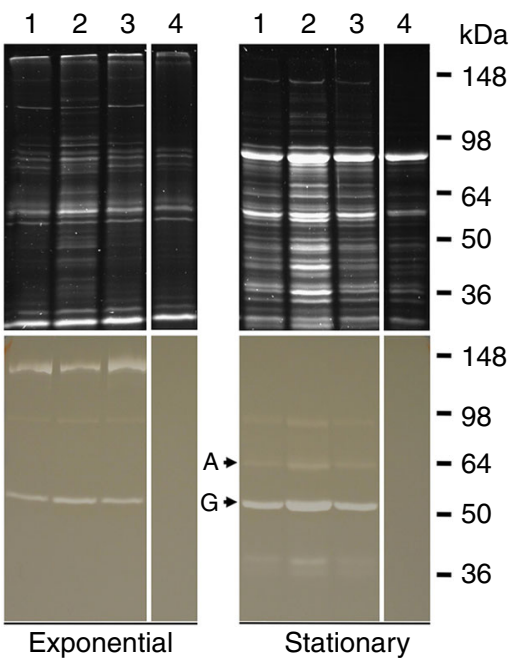

d

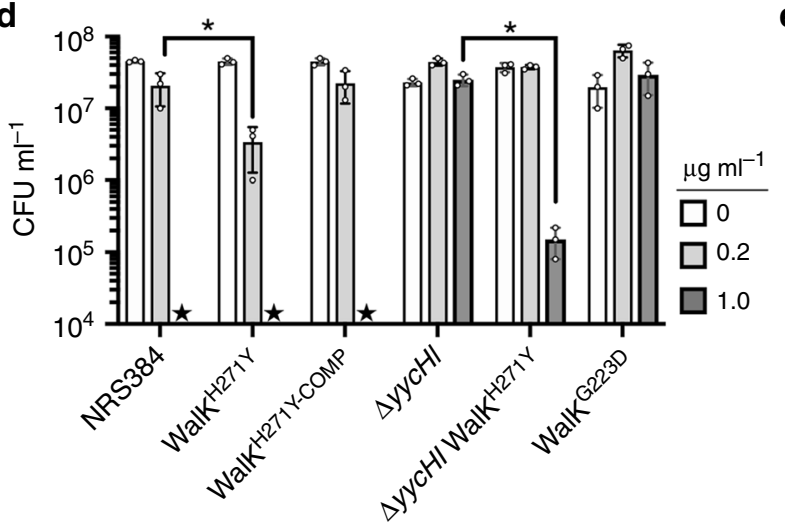

e 0

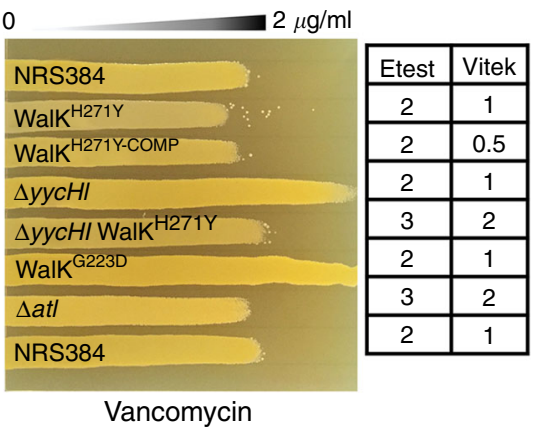

Fig. 2 Phenotypic impact of WalK ${ }^{\mathrm{H}} 271 \mathrm{Y}$ mutation on S. aureus. a Growth kinetics of the WalK ${ }^{\mathrm{H} 271 Y}$ mutant compared to the wild-type (WT) and complemented strains. Overnight cultures were diluted 1:100 into fresh and grown at $37^{\circ} \mathrm{C}(200 \mathrm{rpm})$. The cultures were sampled at the indicated time points for $\mathrm{OD}_{600}$ and colony-forming unit (CFU) readings. The WalK ${ }^{\mathrm{H} 271 Y}$ strain exhibited altered growth kinetics compared to the WT and complemented strains, with the loss of a lag phase and a subsequent reduction in doubling time during exponential growth. Error bars indicate standard deviation (s.d.) from three biological replicates. b Analysis of the total exoproteome and Atl secretion. Supernatant proteins from exponential or stationary phase cultures were run on $10 \%$ sodium dodecyl sulfate-polyacrylamide gel electrophoresis gels for SYPRO Ruby staining or c zymogram analysis through the incorporation of $1.5 \%$ Micrococcus luteus cells. The lane contents are: (1) NRS384, (2) WalK ${ }^{H 271 Y}$, (3) WalK ${ }^{H 271 Y-C O M P}$, and (4) $\Delta a t l$, with 'A' and ' $G^{\prime}$ representing amidase and glucosaminidase, respectively. The protein profile of an at/ mutant was included as a control for Atl activity. $\mathbf{d}$ Impact of the mutations on the sensitivity to lysostaphin. Star denotes 'below the limit of detection' $\left(10^{3} \mathrm{CFU} \mathrm{ml}-1\right)$. Depicted are the data points, mean and s.d. of triplicate biological experiments. The null hypothesis (no difference in mean lysostaphin sensitivity between $\mathrm{WT}$ and $\mathrm{WalK}{ }^{\mathrm{H} 271 \mathrm{Y}}$ at $0.2 \mu \mathrm{g} \mathrm{ml} \mathrm{l}^{-1}$ or $\Delta y y c \mathrm{HI}$ and $\Delta y y c H / W a l K^{H 271 Y}$ at $1 \mu \mathrm{g} \mathrm{ml}^{-1}$ ) was rejected for $P<0.05^{\star}$ (unpaired, Student's $t$ test). e Impact on vancomycin sensitivity. Agar plates were made with a $0-2 \mu \mathrm{g} \mathrm{ml}-1$ concentration gradient of vancomycin. Independent Etest and Vitek vancomycin MIC measurements are indicated next to the gradient plate

(Fig. 2d) with the wild type below the limit of detection. Building on this framework, we then analysed a WalK $\mathrm{G}^{\mathrm{G} 223 \mathrm{D}}$ mutant, which was previously shown to have reduced autophosphorylation/ phosphotransfer between $\mathrm{WalK}$ and $\mathrm{WalR}^{22,23}$. Here this mutant showed increased resistance to lysostaphin, similar to that observed for the $\Delta y y c H I$ mutant (Fig. 2d). We then analysed the impact of the mutations on vancomycin resistance, using antibiotic gradient plates (Fig. 2e). The wild-type, $\mathrm{WalK}^{\mathrm{H} 271 \mathrm{Y}-\mathrm{COMP}}$ and $\Delta a t l$ strains all showed similar levels of resistance. However, the $\Delta y y c H I$ and $\mathrm{WalK}^{\mathrm{G} 223 \mathrm{D}}$ mutant strains showed increased resistance to vancomycin, while the $\mathrm{WalK}^{\mathrm{H} 271 \mathrm{Y}}$ strain exhibited increased sensitivity compared to the wild type. Introduction of $\mathrm{WalK} \mathrm{H}^{\mathrm{H}} 71 \mathrm{Y}$ in the $\Delta y y c H I$ background restored sensitivity to wild-type levels (Fig. 2e). These findings were consistent with Vitek 2 and Etest analyses of the strains (Fig. 2e). Collectively, these results indicate that the $\mathrm{WalK}^{\mathrm{H} 271 \mathrm{Y}}$ mutation activates the WalKR system, resulting in increased sensitivity to lysostaphin and vancomycin.
Structure of the WalK PAS domain. To gain further insight into the impact of the WalK ${ }^{\mathrm{H} 271 \mathrm{Y}}$ mutation, we determined the highresolution structure of the cytoplasmic PAS domain. Domain boundaries for WalK-PAS were defined, based on limited proteolysis, as valine 251 to arginine 376 . This PAS domain sequence (WalK-PAS ${ }^{\mathrm{FULL}}$ ) was cloned and heterologously expressed as a fusion protein with an N-terminal glutathione $S$-transferase (GST) tag and a thrombin cleavage site. WalK-PAS FULL was purified by affinity chromatography, then the affinity tag was removed prior to crystallisation of the PAS domain. A selenomethionine derivative was also generated to aid in phasing the diffraction data (Supplementary Fig. 2). The WalK-PASFULL structure was solved to $2.0 \AA$, although a lack of density for the Nand C-terminus precluded modelling residues 251-262 and 370-376, respectively. Density was also absent for residues 335-338, which represents a disordered loop in WalK-PASFULL. Details of the diffraction data and structure statistics are summarised in Supplementary Table 1. 

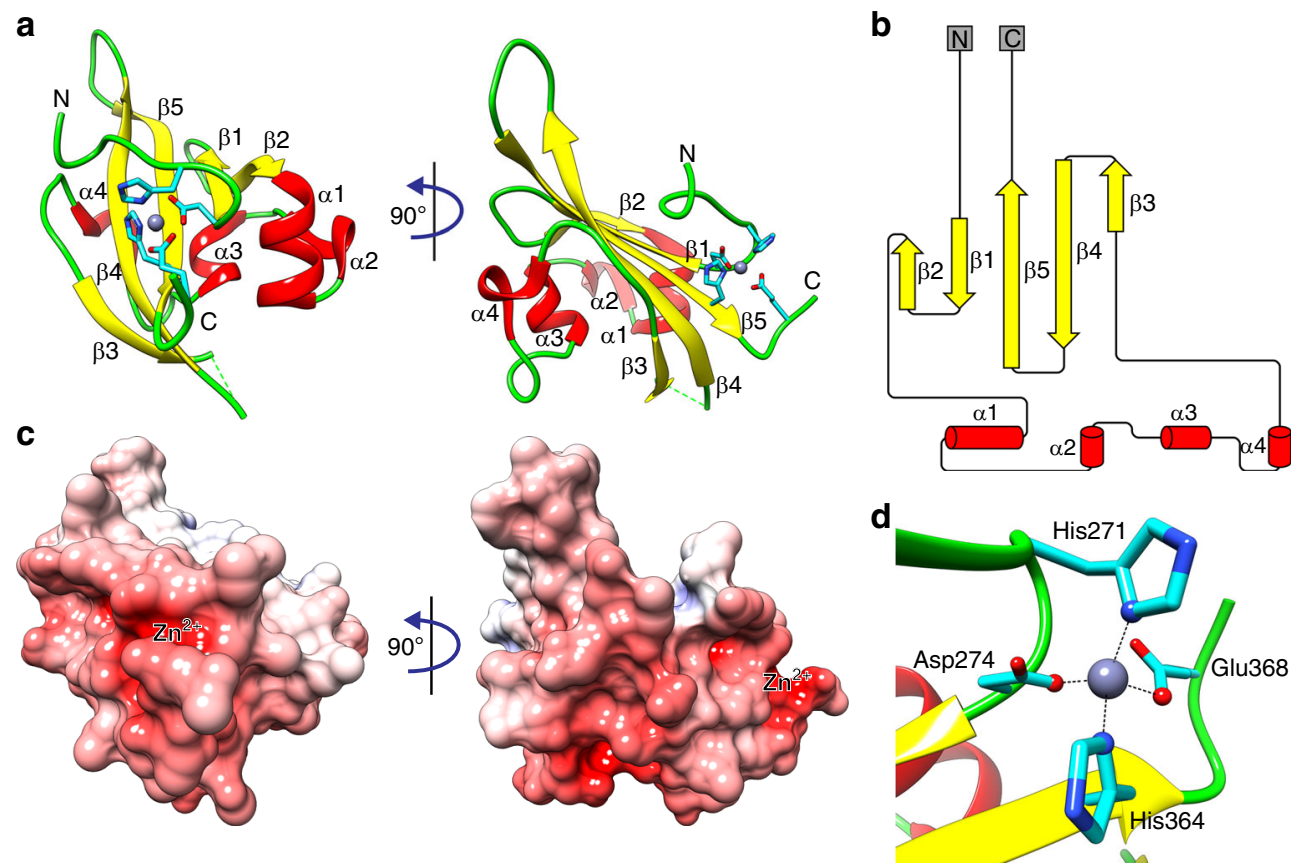

Fig. 3 Crystal structure of S. aureus WalK-PASFULL. a Cartoon representation of the crystal structure of WalK-PASFULL. The $\alpha$-helices are coloured red, $\beta$ strands yellow and loops green. The bound $\mathrm{Zn}^{2+}$ is shown as a grey sphere and its coordinating residues as cyan sticks. The $\mathrm{N}$ - and $\mathrm{C}$-terminus of the structure are labelled. $\mathbf{b}$ Sequence and crystal structure-based secondary structure of WalK-PASFULL generated by Pro-origami66. $\alpha$-Helices are shown as red cylinders and $\beta$-strands as yellow arrows. c Surface electrostatic potential of WalK-PASFULL shown in the same orientation as in a. Positive and negative potentials are shown in blue and red, respectively, coloured continuously between -10 and $10 \mathrm{kT} \mathrm{e}^{-1}$. Surface electrostatic potential was calculated using APBS67; the calculation included the $\mathrm{Zn}^{2+}$ ion. $\mathbf{d}$ The $\mathrm{Zn}^{2+}$-binding site of WalK-PASFULL. Metal-coordinating residues are shown as cyan sticks, with the atoms contributing to the interactions as spheres. The coordinating bonds are illustrated with black dashed lines

The WalK-PAS ${ }^{\text {FULL }}$ structure has a typical PAS domain fold ${ }^{35}$ comprising five antiparallel $\beta$-strands and four $\alpha$-helices, with connecting loops between the helices and $\beta$-strands (Fig. 3a, b). The N-terminal region of the structure is composed of two short $\beta$-strands connected by a small loop. The remainder of the $\mathrm{N}$ terminal region is comprised of four short helices connected by two large loops. The C-terminal portion of the PAS domain predominantly consists of a short $\beta$-strand followed by two larger antiparallel $\beta$-strands connected by a loop. Analysis of the surface electrostatic potential showed an uneven charge distribution on the WalK-PAS ${ }^{\mathrm{FULL}}$ surface (Fig. 3c). In contrast to other PAS domains, well-defined electron density was observed for a single zinc atom coordinated by a hitherto unknown metal-binding site (Fig. 3d). Notably, the $\mathrm{Zn}^{2+}$-binding site resides on the surface of the WalK-PAS FULL domain, with access for $\mathrm{Zn}^{2+}$ from the surrounding solvent. The metal-binding site comprises a single $\mathrm{Zn}^{2+}$ ion bound by the atoms $\mathrm{N} \delta 1$ from His271, O $\delta 1$ from Asp274, N $\delta 1$ from His364 and Oe2 from Glu368 in a slightly distorted tetrahedral coordination geometry (Fig. 3d, Supplementary Fig. 3a). The identity of the bound metal was confirmed to be $\mathrm{Zn}^{2+}$ by the calculation of anomalous difference Fourier maps at energies either side of the $\mathrm{Zn}^{2+}$ absorption edge (Supplementary Fig. 3b, c). The observed bond lengths were $2.2 \AA$, typical for protein coordination of a $\mathrm{Zn}^{2+}$ ion $^{36}$. The $\mathrm{Zn}^{2+}$-binding site, along with the rest of the exposed region, exhibits a negatively charged surface, while the remainder of the WalK-PAS FULL surface is relatively neutral. Although the Nterminal region is well ordered in the structure, the mobility of this region may increase in the absence of the $\mathrm{Zn}^{2+}$ ion and the stabilising interactions conferred by His271 or Asp274.

Analysis of WalK-PAS domain interaction with $\mathrm{Zn}^{2+}$. Further examination of the interaction of the PAS domain with $\mathrm{Zn}^{2+}$ was performed using WalK-PAS ${ }^{\mathrm{FULL}}$ and a truncation mutant (WalKPAS $^{\text {TRUNC }}$ ) in which the unstructured regions (15 residues deleted from the $\mathrm{N}$-terminus and 6 residues from the $\mathrm{C}$-terminus) were removed. The high-resolution structure of WalK-PAS ${ }^{\text {TRUNC }}$ was solved to $2.1 \AA$ (Supplementary Table 1). Comparison of the WalK$\mathrm{PAS}^{\mathrm{FULL}}$ and WalK-PAS ${ }^{\mathrm{TRUNC}}$ structures revealed nearly identical PAS domain folds (Supplementary Fig. 4), with a calculated root mean square deviation (RMSD) of $0.48 \AA$.

To investigate the $\mathrm{Zn}^{2+}$-binding capability of WalK-PAS, we conducted $\mathrm{Zn}^{2+}$-binding assays with both WalK-PASTRUNC and WalK-PAS TRUNC-H271Y, followed by direct metal quantitation by inductively coupled plasma-mass spectrometry (ICP-MS). Zincbinding analyses of WalK-PAS ${ }^{\text {TRUNC }}$ revealed a metal:protein molar ratio of $\sim 0.3: 1$, suggesting that WalK-PAS was $\sim 30 \% \mathrm{Zn}^{2+}$ bound at any given time, whereas no $\mathrm{Zn}^{2+}$ binding was observed for WalK-PAS ${ }^{T R U N C-H 271 Y}$. Taken together, these data indicate that the H271Y mutation abolishes $\mathrm{Zn}^{2+}$ binding by the WalK PAS domain, supporting the inference that metal binding at this site influences WalK activity. We then determined the affinity of $\mathrm{Zn}^{2+}$ for WalK-PAS ${ }^{\mathrm{FULL}}$, which revealed a moderate affinity interaction with a $K_{\mathrm{D}}$ of $0.503 \pm 0.03 \mu \mathrm{M}$ (Supplementary Fig. 5a). Taken together, these data suggest that $\mathrm{Zn}^{2+}$ is unlikely to serve a structural role in the WalK-PAS domain, since structural $\mathrm{Zn}^{2+}$ sites in proteins are typically formed by coordination spheres comprising $\mathrm{Cys}_{4}$ or $\mathrm{Cys}_{2}$-His-Cys ligands and they are associated with much higher binding affinities for $\mathrm{Zn}^{2+}(100 \mathrm{nM}-100 \mathrm{pM})^{36}$. In contrast, Glu and Asp residues are infrequently found in structural $\mathrm{Zn}^{2+}$ sites, with a recent analysis of NCBI Protein Data Bank noting a prevalence of $\leq 6.0 \%$ for relevant protein structures ${ }^{36}$. Collectively, these data, in combination with the phenotypic observations for the WalK ${ }^{\mathrm{H} 271 \mathrm{Y}}$ mutant, strongly suggest that the metal binding site in WalK-PAS serves a regulatory role than a structural function. 
PAS-domain $\mathrm{Zn}^{2+}$ binding regulates WalK autophosphorylation. To elucidate the potential regulatory role of metal binding in the WalK-PAS domain, we first investigated the relevance of $\mathrm{Zn}^{2+}$ interaction to the cytoplasmic PAS domain. This was addressed by determining the cellular quotient of $\mathrm{Zn}^{2+}$ ions via the combination of electron microscopic measurements of S. aureus, enumeration of CFUs at a fixed cell density and ICPMS analyses to derive the intracellular metal ion accumulation data in molar units. S. aureus was determined to have a mean cell volume of $0.26 \pm 0.07 \mathrm{fl}(n=37)$ and a derived concentration of $\mathrm{Zn}^{2+}$ ions of $3.27 \pm 0.23 \mathrm{mmol}^{-1}$ (Supplementary Fig. 5b). Although this concentration represents the total abundance of cellular $\mathrm{Zn}^{2+}$ ions, comprising both tightly bound metal-protein complexes and labile pools of exchangeable ions, the concentration of $\mathrm{Zn}^{2+}$ is significantly greater than the observed $K_{\mathrm{D}}$ for the WalK-PAS domain. Thus cellular $\mathrm{Zn}^{2+}$ abundance is within a plausible range for interaction with WalK. We then examined the impact of the H271Y mutation on WalK autophosphorylation activity in vitro. Measurements were performed using the cytoplasmic domains of WalK ${ }^{208-608}$ (WalK ${ }^{\mathrm{CYTO}}$ ), which has previously been studied using autophosphorylation assays ${ }^{10,23}$. Here we observed that the H271Y mutation (WalKCYTO-H271Y) resulted in a significant, $~ 50 \%$ increase in autophosphorylation, by comparison to WalK ${ }^{\mathrm{CYTO}}(p<0.01$, Mann-Whitney $U$ test, Fig. 4a). Hence, these data indicate that $\mathrm{Zn}^{2+}$ binding by the PAS domain negatively regulates the autophosphorylation activity of WalK in vitro, consistent with the altered WalK activity observed by in vivo phenotypic analyses.

To complement the in vitro findings, we investigated the in vivo phosphorylation status of WalR. We first established the detection efficiency for the labile aspartic acid phosphorylation on residue D53 of WalR. This was achieved by constructing two WalR variants in the tetracycline-inducible plasmid pRAB11, containing either (i) native WalR with a $3 \times F L A G$ tag on the C-terminus (WalR-FLAG) or (ii) a mutant variant of WalR-FLAG, wherein mutation of D53 to an alanine (D53A). The D53A mutation abolishes the potential for phosphorylation at this site. The above plasmids along with empty pRAB11 were transformed into the $S$. aureus NRS384 wild-type, WalK ${ }^{\mathrm{H} 271 \mathrm{Y}}, \Delta p k n B$ (serine threonine kinase) and $\Delta \operatorname{stp} 1$ (serine threonine phosphatase). The latter two strains were included to differentiate the site of phosphorylation. PknB has been shown to phosphorylate WalR at residue T101 ${ }^{37}$, while in B. subtilis, the deletion of Stp1 led to increased WalR phosphorylation at the site equivalent to WalR ${ }^{\mathrm{T} 101} 38$. Here we observed that D53 phosphorylation on WalR ${ }^{\mathrm{FLAG}}$ was absent in

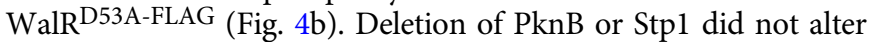
D53 phosphorylation, indicating that WalK was the dominant contributor. Further, our analyses did not detect a second site of phosphorylation under these conditions, suggesting that T101 was not being phosphorylated or detected under the conditions tested. Increased phosphorylation of WalR was observed in the WalK ${ }^{\mathrm{H} 271 Y}$ background (Fig. 4b), consistent with the autophosphorylation results (Fig. 4a). Building on this framework, we examined D53 phosphorylation in the native context using chromosomally tagged strains (WalR ${ }^{\mathrm{FLAG}}$ ) in either wild-type or WalK ${ }^{\mathrm{H} 271 \mathrm{Y}} S$. aureus. The ability to modify the chromosomal copy of walR showed that the FLAG tag did not dramatically alter WalR activity. When analysed throughout growth, there was a striking increase in the phosphorylation of WalR in the WalK ${ }^{\mathrm{H} 271 \mathrm{Y}}$ strain over the wild type from mid-log phase onwards, with the difference exaggerated as growth progressed into late log/ early stationary phase (Fig. 4c, d). These findings are consistent with the WalK autophosphorylation assay (Fig. 4a) and show that the negative regulation of WalKR imposed by $\mathrm{Zn}^{2+}$ binding is important in dampening the response of WalK as the cells transition out of active growth.
We then measured the impact of exogenous $\mathrm{Zn}^{2+}$ on expression of WalR-controlled genes in mid-exponential phase $S$. aureus. To do this, we constructed a fluorescence-based promoter reporter using the well-described WalR-regulated gene is $a A$, encoding a lytic transglycosylase ${ }^{10}$. We first verified WalR-specific control of isaA by mutation of the published WalR-binding motif (Supplementary Fig. 1b) and identified that WalR positively regulates isaA (Fig. $4 \mathrm{e})^{10}$. We predicted that exposure of $S$. aureus to $\mathrm{Zn}^{2+}$ would reduce WalR binding to the is $A$ A promoter and thus decrease fluorescence. Growth of the isaA reporter strain to mid-log phase in the presence of trace $\mathrm{Zn}^{2+}$ concentrations (Chelex100 -treated $\mathrm{RPMI}<300 \mathrm{nM} \mathrm{Zn}^{2+}$ ) and then exposed to $25 \mu \mathrm{M}$ $\mathrm{Zn}^{2+}$ led to a significant reduction in fluorescence $(p<0.05$, two-way analysis of variance (ANOVA), Fig. 4f). Chelation of the $\mathrm{Zn}^{2+}$ with TPEN significantly relieved isaA promoter repression $(p<0.05$, two-way ANOVA, Fig. $4 \mathrm{f})$. As predicted, the WalK $\mathrm{K}^{\mathrm{H} 271 \mathrm{Y}}$ mutant was unresponsive to the presence of either $\mathrm{Zn}^{2+}$ or $\mathrm{Zn}^{2+}+$ TPEN. Collectively, these data demonstrate that WalK ${ }^{\mathrm{PAS}-\mathrm{CYT}}$ is an intracellular sensor that potentiates the WalR regulon via interaction with $\mathrm{Zn}^{2+}$ ions.

Metal-induced conformational changes in WalK. To further understand the structural consequences of $\mathrm{Zn}^{2+}$ binding by WalK and the impact on kinase activity, we employed molecular dynamics (MD) (Supplementary Fig. 6). MD simulations indicated that $\mathrm{Zn}^{2+}$ binding directly influences the relative positioning of the PAS and catalytic (CAT) domains. In the absence of $\mathrm{Zn}^{2+}$, the dihedral angle between the PASCYTO and CAT domains in each monomer was $\sim 136^{\circ}$ when viewed down the central axis of the kinase (measured as the dihedral angle between Ca atoms of residues 288, 271, 369 and 569, averaged between the two chains), while the average distance between the upper and lower helices of the cytoplasmic domains (measured from the $\mathrm{Ca}$ atoms of residues 271 and 359 and averaged between the two chains) was $21.6 \AA$ (Fig. 5a). In the presence of $\mathrm{Zn}^{2+}$, the relative dihedral angle increased to $175^{\circ}$ while the intra-helical distance decreased to an average of $12.3 \AA$ (Fig. $5 \mathrm{~b}$ ). The binding of the metal ion also stabilises the PAS domain fold by bringing the $\mathrm{N}$ and C-terminal regions into closer proximity. This is particularly evident for the N-terminal H271 and the C-terminal Glu368 residues. Collectively, our structural analyses suggest that metal binding in the PAS domain results in significant conformational changes. These metal-induced structural rearrangements provide a mechanistic basis for how conformational changes arise in WalK to negatively regulate its autokinase activity and subsequent signal transduction to the response regulator, WalR.

Conservation of metal-binding sites in WalK within the Firmicutes. We then examined the potential conservation of the WalK PASCYTO domain metal-binding site across low $\mathrm{G}+\mathrm{C}$ Gram-positive bacteria. Alignment of a selection of WalK proteins from different genera with the $S$. aureus WalK reference sequence revealed that $\mathrm{H} 271$ is conserved among the coagulasepositive/coagulase-negative staphylococci and enterococci, but not in streptococci or listeria, where it is replaced by a tyrosine residue (Fig. 6a). A Y271 residue was also present in two out of the five bacilli examined, including the well-studied strain, $B$. subtilis 168. Notably, the three additional metal-coordinating residues were only conserved among staphylococci and enterococci, with all other genera having at least one deviation from the $S$. aureus consensus. These results raise the testable hypothesis that WalK metal binding is restricted to staphylococci and enterococci and might enable additional regulatory control of the essential WalKR TCS in those genera. We next examined the 


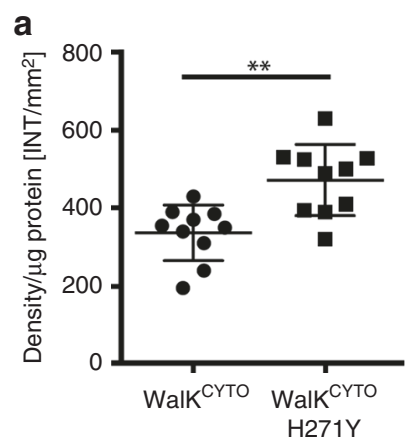

C

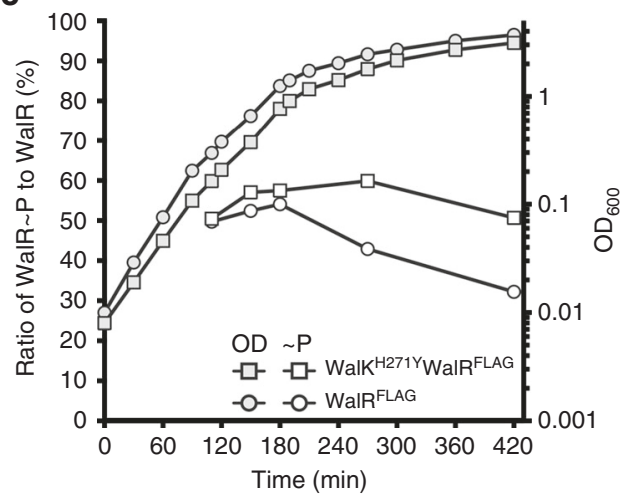

b

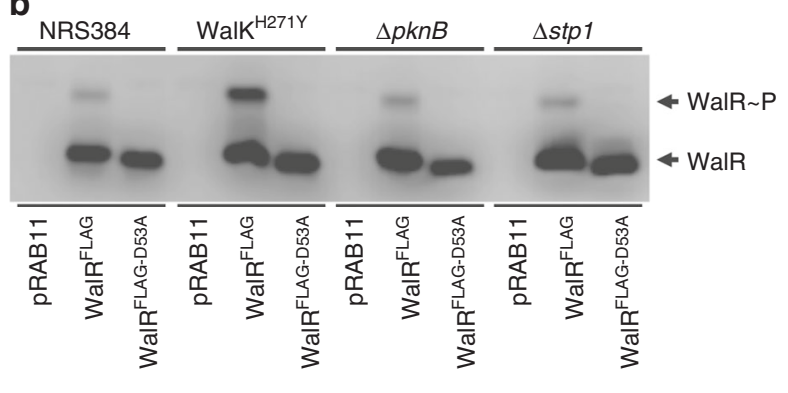

d

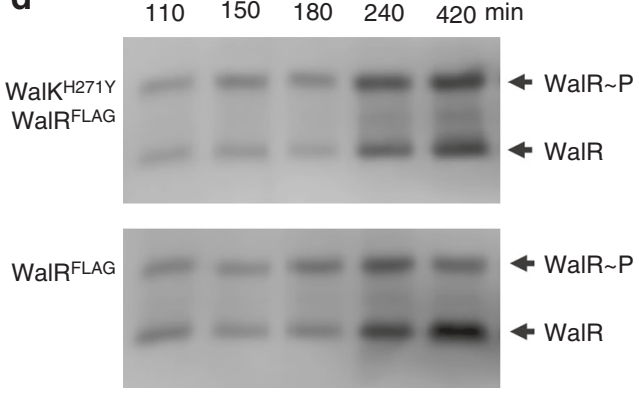

e

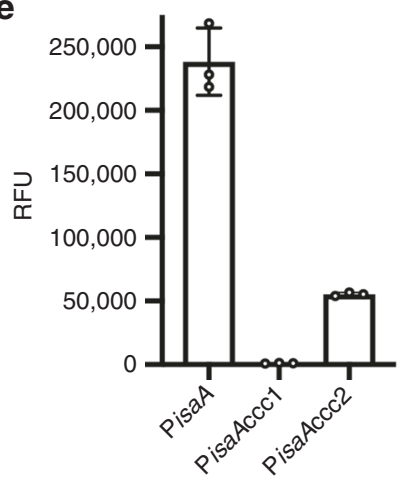

$\mathbf{f}$

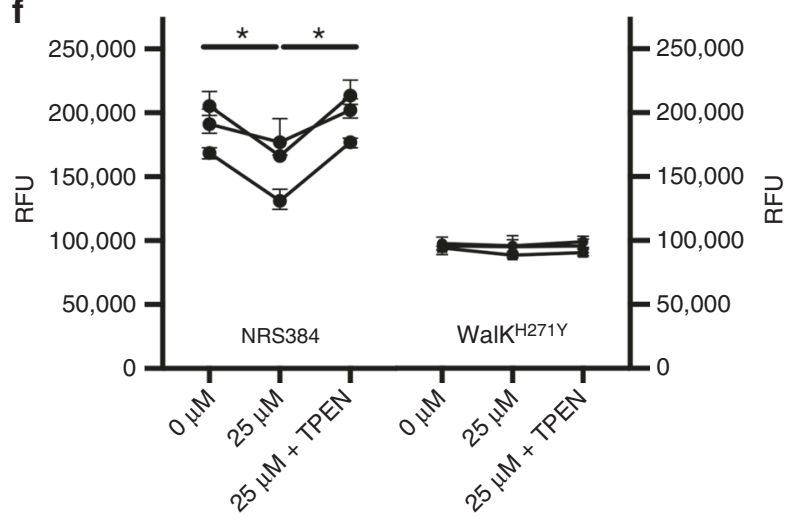

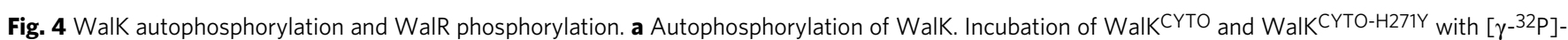
ATP for 60 min shows that H271Y mutation significantly increases WalK autophosphorylation ( ${ }^{\star \star} p=0.0016, n=10$, error bars s.d.). Null hypothesis (no difference between means) was rejected for $p<0.01$ (two-tailed Mann-Whitney $U$ test). b Establishing phos-tag acrylamide for the analysis of WalR phosphorylation. Either pRAB11 (empty vector), WaIRFLAG (wild-type WalR with 3×FLAG tag) or WalRD53A-FLAG (mutation to abolish D53 phosphorylation in WalR ${ }^{F L A G}$ ) were transformed into NRS384, WalK ${ }^{\mathrm{H} 271 Y}$ and $\Delta p k n B$ or $\Delta$ stp1. WalR was detected by western blot with anti-FLAG M2 monoclonal antibody. c Analysis of chromosomally FLAG-tagged WalR by phos-tag. The walR gene in either the wild-type or WalK ${ }^{\mathrm{H} 271 Y}$ background was tagged with a $3 \times$ FLAG tag on the C-terminus. A growth profile (grey circle/square) and the phosphorylation status (white circle/square) of WalR was examined at time points equating to early, mid, late log and early stationary phase. The ratio of phosphorylated to non-phosphorylated WalR presented in $\mathbf{c}$ was determined by densitometry of phos-tag electrophoresed/western blotted samples shown in d. e Mutation of WalR-binding sites to confirm that PisaA is regulated by WalR. Cumulative fluorescence expression from the native isaA promoter region or isaA with mutated WalR-binding sites (CCC 1 or CCC 2, Supplementary Fig. 1B) were introduced into the enhanced yellow fluorescent protein reporter plasmid and transformed into NRS384. Strains were grown in Luria Broth to stationary phase with the level of fluorescence determined from three independent experiments. $\mathbf{f} I m p a c t$ of $\mathrm{Zn}^{2+}$ on the expression of isaA in metal defined media, with the addition of (i) $0 \mu \mathrm{M} \mathrm{ZnSO}_{4}$, (ii) $25 \mu \mathrm{M} \mathrm{ZnSO}_{4}$, or (iii) $25 \mu \mathrm{M} \mathrm{ZnSO} 4$ and $10 \mu \mathrm{M}$ TPEN to S. aureus NRS384 grown to stationary phase $(24 \mathrm{~h})$ with the level of fluorescence determined from three independent experiments (all shown). Depicted are the individual data points, mean and s.d. from these three replicates. The null hypothesis (no difference between means) was rejected for $p<0.05$ (two-way analysis of variance with Tukey correction, $\left.{ }^{\star} p<0.05\right)$. Source data are provided as a Source Data file

structural alignment of the WalK-PASCYT domains of $S$. aureus

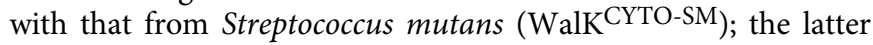
natively encodes the Y271 substitution and was crystallised in the context of the complete cytoplasmic domain ${ }^{39}$ (Fig. 6b). Although the two PASCYTO domains align with a relatively large RMSD of $2.31 \AA$, indicating significant structural differences, the largest deviations occur in the regions comprising the $\mathrm{Zn}^{2+}$-binding site of the $S$. aureus PASCYTO domain. In $S$. mutans, the WalKCYTO-SM domain forms a leucine zipper dimeric interface with an adjacent monomer. Metal binding by $S$. 
aureus WalK-PASCYTO is predicted to preclude formation of such an interface. The $\mathrm{Zn}^{2+}$ atom would be positioned near the centre of the structure, resulting in steric clashes at the dimeric PAS ${ }^{C Y T O}$ domain interface that would likely impede WalK dimer interactions (Fig. 6c).

\section{Discussion}

In this study, we provide evidence of a specific ligand for the WalKR system, opening new avenues to understand the function and essentiality of this regulon. The $S$. aureus WalKR TCS was

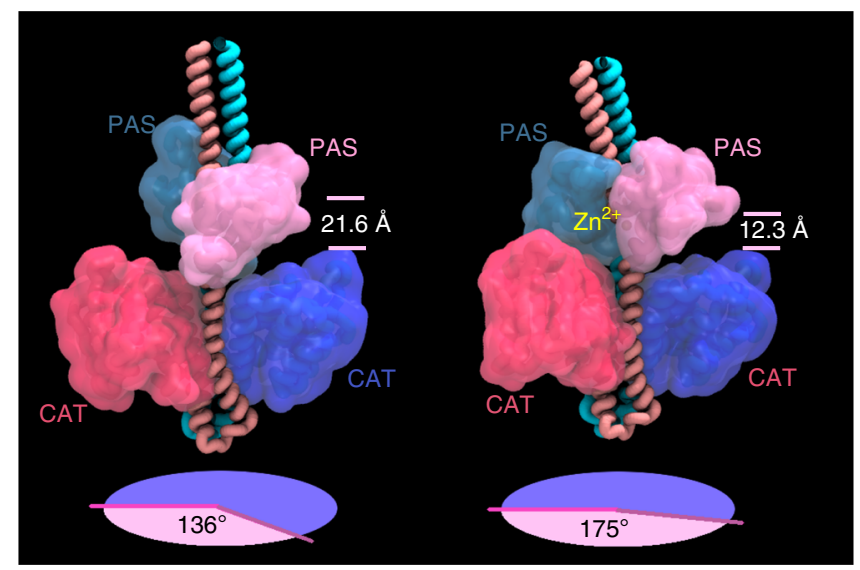

Fig. 5 Molecular modelling of WalK in the presence or absence of $\mathrm{Zn}^{2+}$. Still images taken from molecular dynamics simulation of full-length, membrane-bound S. aureus WalK in the $\mathbf{a}$ absence and $\mathbf{b}$ presence of $\mathrm{Zn}^{2+}$, showing the predicted conformational changes induced in the WalK PerArnt Sim (PAS) and catalytic (CAT) domain upon metal binding. Dihedral angles between the cytoplasmic PAS and CAT domains were measured over the course of the simulations (lower circular projections) and suggested that $\mathrm{Zn}^{2+}$ binding directly influences the relative positioning of the PAS and CAT domains. In the absence of $\mathrm{Zn}^{2+}$, the dihedral angle between the PAS ${ }^{\text {CYTO }}$ and CAT domains in each monomer was $136^{\circ}$ when viewed down the central axis of the kinase, while the average distance between the upper and lower helices of the cytoplasmic domains was 21.6 $\AA$. In the presence of $\mathrm{Zn}^{2+}$, this angle changed to $\sim 175^{\circ}$ and the average distance between the upper and lower helices contracted to $12.3 \AA$ identified in the late 1990s, but the ligand(s) sensed by this HK and subsequent mechanisms of activation have remained elusive. One proposed model for WalK sensing has been through the recognition of the D-Ala-D-Ala moiety of Lipid II via the extracytoplasmic PAS domain ${ }^{6}$. This molecule would be abundant at the site of septation during exponential growth but become limiting upon the cessation of cellular replication. Although this scenario provides a link between activation of autolysin production, via phosphorylation of WalR, with division septum localisation of WalK, there remains a paucity of experimental evidence to support this model ${ }^{40}$. Here we have shown that the presence of a metal-binding site within the PASCYTO domain of WalK directly influences the activation status of the protein. Abrogation of in vitro $\mathrm{Zn}^{2+}$-binding capacity of WalK increased the autophosphorylation of WalK and in vivo phosphotransfer to WalR. There are several key examples of regulation of $\mathrm{HK}$ activity via PAS domain ligand binding, such as oxygen sensing by Bradyrhizobium japonicum FixL, wherein haeme binding by the cytoplasmic PAS domain regulates nitrogen fixation under reduced oxygen tensions ${ }^{35}$; oxygen sensing by Staphylococcus carnosus NreB, in which the PASCYTO domain contains an oxygen-labile iron-sulfur cluster ${ }^{41}$; and redox status sensing by Azotobacter vinelandii NifL, where the PAS ${ }^{\mathrm{CYTO}}$ domain binds nicotinamide adenine dinucleotide ${ }^{42}$. Direct metal ion binding has previously been observed in extracellular PAS domains, such as PhoQ from Salmonella typhimurium, which senses the cations $\mathrm{Ca}^{2+}$ and $\mathrm{Mg}^{2+, 43}$. However, to the best of our knowledge, metal binding by a cytoplasmic PAS domain has not previously been reported and appears to be a highly restricted attribute among the staphylococci and enterococci, despite WalK being conserved among the Firmicutes. For instance, the WalK PASCYTO domain in S. mutans has a naturally occurring tyrosine at residue 271 and structural analysis shows no evidence of metal binding ${ }^{39}$.

Recapitulation of the walk $\mathrm{H} 271 \mathrm{Y}$ mutation in vivo resulted in phenotypes associated with activation of WalK (e.g. sensitivity to lysostaphin and vancomycin, increased haemolysis and production of the major autolysin Atl) along with the loss of lag phase upon inoculation into fresh media. This latter phenotype would be consistent with the requirement for accumulation of a ligand sensed by the extracellular PAS domain leading to the activation of WalKR and subsequent autolysin production. By contrast with B. subtilis, YycHI are activators of the S. aureus WalK system ${ }^{28,29}$. a

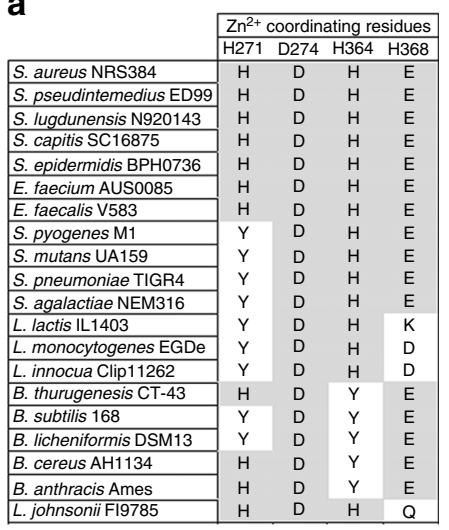

b

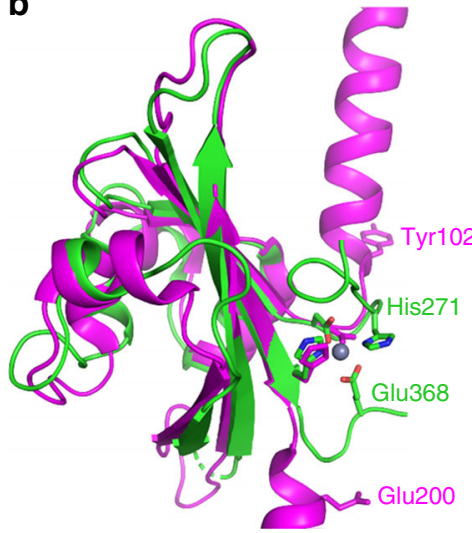

C

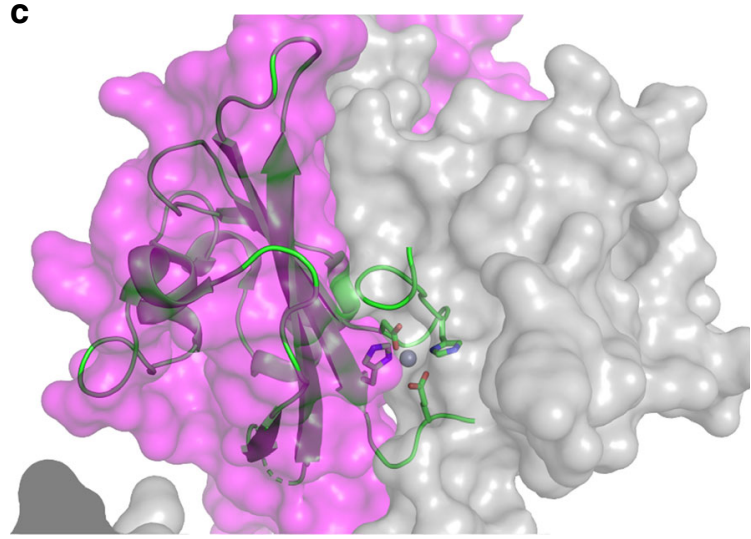

Fig. 6 Structural comparison of WalK. a Comparison of metal-coordinating residues from a range of Gram-positive bacteria with WalK orthologues. Protein sequences were aligned with ClustalW using default parameters. Residues that match the S. aureus consensus are highlighted in grey. $\mathbf{b}$ Superposition of the crystal structure of WalK-PASFULL (green) with VicK (magenta, PDB: 4I5S) in cartoon representation. The $\mathrm{Zn}^{2+}$-coordinating residues in WalKPASFULL are shown as sticks, with the homologous residues in VicK shown in magenta. The bound $\mathrm{Zn}^{2+}$ ion is shown as a sphere. c Superposition of WalKPASFULL (green) with the $\mathrm{VicK}_{2}$ homodimer shown by transparent surface representation (magenta/grey, PDB: 4I5S). The crystal structure of WalKPASFULL is shown by cartoon representation with the $\mathrm{Zn}^{2+}$-binding site metal-coordinating residues shown as sticks and the bound ion as a sphere 
Consequently, the metal-dependent regulation of the WalK-PAS domain in S. aureus may serve as a dynamic constraint on kinase activity. Additional levels of regulation of the WalKR operon have also been identified. These include a second site of phosphorylation, residue T101 on WalR, by the serine threonine kinase known as Stk1 or $\mathrm{PknB}^{37}$ and the interaction of SpdC (previously called lysostaphin resistance factor A-lyrA $)^{44}$ with WalK, which negatively regulate genes under the control of $\mathrm{WalR}^{45}$. Both PknB and SpdC are localised to the division septum similar to WalK ${ }^{31,37}$, highlighting the complexity of this regulatory axis. The establishment of phos-tag acrylamide to analyse the phosphorylation status of WalR in vivo is a powerful tool to investigate the regulation of this essential system (Fig. 4).

The PASCYTO domain of S. aureus WalK binds $\mathrm{Zn}^{2+}$ with only moderate affinity, suggesting a regulatory role for metal binding rather than an obligate structural function. In this manner, it is possible that this site only has transient interactions with $\mathrm{Zn}^{2+}$ thereby facilitating a continuum of WalK activation states rather than serving as a binary switch. Disruption of PASCYTO domain dimerisation upon metal binding could impact WalK activity in a similar manner to the L100R mutation in Streptococcus pneumoniae $^{46}$. The L100R mutation destabilises S. pneumoniae PAS dimerisation, leading to a loss of autophosphorylation activity. Our structural analyses and molecular dynamics simulations suggest that $\mathrm{Zn}^{2+}$ binding induces large conformational changes that alter the relative positions of the PASCYTO and catalytic domains (Fig. 5). These observations provide a plausible mechanistic basis for the reduction in WalK function, although the precise mechanism remains to be elucidated.

Intriguingly, although the amino acid sequence differences between WalK from the staphylococci and enterococci compared to other Firmicutes are small, these differences may have major functional consequences. Our observations suggest that there may be additional intracellular regulator control of WalK. Metal ion abundances can change significantly during bacterial infection, and in this way, sensing these fluxes could enable rapid adaptation to distinct host niches. Alternatively, these data may suggest that WalK has additional or differing roles in regulation beyond peptidoglycan biosynthesis in these genera. Potential regulatory roles for WalK may include contributing to maintenance of intracellular metal homeostasis, such as sensing of intracellular $\mathrm{Zn}^{2+}$ abundance by WalK. However, we note that transcriptomic analyses of $S$. aureus WalKR mutants predominantly highlight genes and pathways involved in nucleotide metabolism, rather than those associated with metal ion transport ${ }^{22,26}$. Nonetheless, indirect metal-dependent regulation could influence such pathways indirectly. One possible mechanism could be via Stp1, a $\mathrm{Mn}^{2+}$-dependent phosphatase, and its cognate kinase PknB, which interacts with WalKR and influences its activity. Further insight into the role of metal coordination in regulating this HK is necessary to understand the essentiality of the system in S. aureus.

\footnotetext{
Methods

Strains, primers, reagents and media. Bacterial strains/plasmids and primers (IDT) used are described in Supplementary Table 2 and Supplementary Table 3, respectively. S. aureus were routinely grown on BHI agar (Difco), TSB (Oxiod) or Luria Broth (LB) at $37^{\circ} \mathrm{C}$ with shaking at $200 \mathrm{rpm}$. For the selection of pIMAY-Z or pIMC8-YFP containing strains, BHI agar was supplemented with $10 \mu \mathrm{g} \mathrm{ml}^{-1}$ chloramphenicol and $100 \mu \mathrm{g} \mathrm{m}^{-1} \mathrm{X}$-gal (BHIA-CX). For the protein expression, Terrific Broth (TB) was used $\left(10 \mathrm{gl}^{-1}\right.$ tryptone, $24 \mathrm{gl}^{-1}$ yeast extract, $10 \mathrm{gl}^{-1}$ glucose, $0.17 \mathrm{M} \mathrm{KH}_{2} \mathrm{PO}_{4}$ and $0.72 \mathrm{M} \mathrm{K}_{2} \mathrm{HPO}_{4}$ ). Restriction enzymes, Phusion DNA polymerase and T4 DNA ligase were purchased from New England Biolabs. Phire Hotstart II (for colony PCR) was purchased from Thermo Fisher. Genomic DNA from S. aureus was isolated from $1 \mathrm{ml}$ of an overnight culture (DNeasy Blood and Tissue Kit-Qiagen) pretreated with $100 \mu$ g of lysostaphin (Sigma cat. no. L7386). Lysostaphin sensitivity assays were performed as described ${ }^{32}$.
}

S. aureus site-directed mutagenesis by allelic exchange. The walk $\mathrm{K}^{\mathrm{H}} \mathrm{H} 1 \mathrm{Y}$ mutation was recombined into the chromosomal copy of walK in the USA300 background (NRS384) by allelic exchange. The region encompassing walK was amplified by SOE-PCR to introduce a neutral change in the third nucleotide of codon 270 and the first nucleotide in codon 271 with primer set IM7/IM8/IM9/ IM10 (Supplementary Table 3). The resultant $2.7 \mathrm{~kb}$ product was recombined into pIMAY-Z by the seamless ligation cloning extract (SLiCE) method ${ }^{32,47}$ and transformed into Escherichia coli $\mathrm{IM} 08 \mathrm{~B}^{48}$. The sequence-confirmed construct was extracted from E. coli, ethanol precipitated and transformed into electrocompetent NRS384 ${ }^{49}$. Allelic exchange was performed as described ${ }^{48}$, with the mutation screened using primer pair IM11/IM12 with an annealing temperature of $65^{\circ} \mathrm{C}$. Reversion of the WalK ${ }^{\mathrm{H} 271 \mathrm{Y}}$ mutation to WalK ${ }^{\mathrm{H} 271 \mathrm{Y}-\mathrm{COMP}}$ was achieved through allelic exchange in NRS384 WalK ${ }^{\mathrm{H} 271 \mathrm{Y}}$. A marked walK allele was constructed through the introduction of a silent PstI site (walK nucleotide 1302, A to G) into walK by SOE-PCR with primer set IM7/IM58/IM59/IM10. A 3×FLAG tag was introduced onto the C-terminus of WalR by SOE-PCR with primers IM31/IM108/ IM109/IM40. Deletion of genes or insertion of a FLAG tag was performed by SOEPCR with the product cloned into pIMAY-Z by SLiCE with the following primer sets: $\Delta a t l$ gene (IM96/IM97/IM98/IM99: entire gene), $\Delta y y c H I$ (IM54/IM78/IM79/ IM75: from codon 5 of $y y c H$ to the stop codon of $y y c I$ ), $\Delta s t p 1$ (IM251/IM252/ IM253/IM254: from the start codon leaving the last 8 amino acids), $\Delta p k n B$ (IM255/ IM256/IM257/IM258: entire gene), or walR ${ }^{\mathrm{FLAG}}$ (IM31/IM108/IM109/IM40). The walK $^{\mathrm{G} 223 \mathrm{D}}$ mutation was directly amplified by PCR on JKD6008 genomic DNA with IM7/IM10 (Supplementary Table 3). The purified PIMAY-Z construct was transformed into NRS384 after passage through IM08B (Supplementary Table 3) Allelic exchange was performed as described above. To screen for the walk ${ }^{\mathrm{FLAG}}$, IM111/IM181 was used to identify the insertion. For WalK ${ }^{\mathrm{G} 223 \mathrm{D}}$ after allelic exchange, white colonies were screened for decreased sensitivity to vancomycin. Genome sequencing and analysis of the isolates was conducted as described, with paired-end libraries prepared using NexteraXT and sequenced on an Illumina NextSeq ${ }^{32}$. Resulting reads were mapped to a NRS384 reference genome and mutations identified using Snippy (https://github.com/tseemann/snippy) ${ }^{32}$.

Construction of anhydrotetracycline (ATc)-inducible WalR-FLAG. The walRFLAG gene was PCR amplified from NRS384 walk ${ }^{\mathrm{FLAG}}$ genomic DNA with primers IM280/IM305 (wild type) or by SOE-PCR with primers IM280/IM278/IM279/ IM305 to introduce the walR $R^{\mathrm{D} 53 \mathrm{~A}}$ mutation. The products were digested with $\mathrm{KpnI} /$ BamHI and ligated into KpnI/BglII-digested pRAB11. Ligations were transformed into IM08B to produce either pRAB11 walR ${ }^{\text {FLAG }}$ or $\mathrm{pRAB} 11$ wal $R^{\mathrm{D} 53 \mathrm{~A}-\mathrm{FLAG}}$. Plasmids (pRAB11, pRAB11walR $R^{\mathrm{FLAG}}$ or pRAB11 walR ${ }^{\mathrm{D} 53 \mathrm{~A}-\mathrm{FLAG}}$ ) were transformed into NRS384, NRS384walk ${ }^{\mathrm{H} 271 Y}$, NRS384 $\Delta$ stp1 or NRS384 $\Delta p k n B$ and selected on BHIA-CX at $37^{\circ} \mathrm{C}$.

\section{Construction of a PisaA-YFP and Patl-YFP fusion to monitor WalR-dependent} activation. The $S$. aureus codon-optimised enhanced yellow fluorescent protein (YFP) and upstream TIR sequence from pYFP- $\mathrm{F}^{50}$ were PCR amplified with primers IM314/IM315. The product was digested with $\mathrm{KpnI} / \mathrm{SacI}$ and cloned into the complementary digested pIMC $^{48}$, creating pIMC8-YFP. To clone into pIMC8YFP, the vector was digested with KpnI, gel extracted and PCR amplified with IM1/ IM385. The PCR purified vector and gel extracted PisaA (IM363/IM364-600 bp) or Patl (IM96/IM360) were combined, SLiCE cloned and then transformed into E. coli IM08B, with selection on Luria agar plates containing chloramphenicol $10 \mu \mathrm{g} \mathrm{ml}^{-1}$ Plasmids were extracted and directly transformed by electroporation into NRS384 or NRS384 WalK ${ }^{\mathrm{H} 271 Y}$. Mutations were introduced into the two WalR-binding motifs (TGTWAH $\left(\mathrm{N}^{5}\right)$ TGTWAH $\rightarrow$ CCCWAH $\left(\mathrm{N}^{5}\right)$ TGTWAH) in isaA by SOEPCR with primer sets CCC-1 (49nt up from the transcription start site (TSS) IM363/IM1123; IM1122/IM364) or CCC-2 (228nt up from the TSS-IM363/ IM1125; IM1124/IM364). These products were cloned into pIMC8-YFP as described above. The expression from pIMC8-YFP, pIMC8-PisaA-YFP, pIMC8-

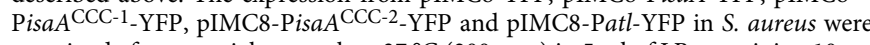
examined after overnight growth at $37^{\circ} \mathrm{C}(200 \mathrm{rpm})$ in $5 \mathrm{ml}$ of $\mathrm{LB}$ containing $10 \mu \mathrm{g}$ $\mathrm{ml}^{-1}$ of chloramphenicol in a $50 \mathrm{ml}$ Falcon tube. Each strain was read in triplicate on the Ensight multimode plate reader (PerkinElmer) with 100 flashes of excitation at $512 \mathrm{~nm}$ and emission detection at $527 \mathrm{~nm}$. The $S$. aureus strains NRS384 or NRS384 walK $^{\mathrm{H} 271 \mathrm{Y}}$ containing pIMC8-YFP or pIMC8-PisaA-YFP were grown overnight at $37^{\circ} \mathrm{C}(200 \mathrm{rpm})$ in $5 \mathrm{ml}(50 \mathrm{ml}$ Falcon tube) of complex Chelex-100treated RPMI (containing $1 \mu \mathrm{M} \mathrm{MgCl}_{2}, 0.1 \mu \mathrm{M} \mathrm{CaCl}_{2}, 1 \mu \mathrm{M} \mathrm{FeCl}_{2}$ and $1 \%$ Casamino acids, $10 \mu \mathrm{g} \mathrm{ml}^{-1}$ chloramphenicol). The abundance of transition metal ions in Chelex-100-treated culture media was determined by ICP-MS and $\mathrm{Zn}^{2+}$ confirmed to be $<300 \mathrm{nM}$. The cultures were diluted $1: 100$ in $10 \mathrm{ml}$ of prewarmed Chelex-100-treated RPMI $\left(1 \mu \mathrm{M} \mathrm{MgCl}_{2}, 0.1 \mu \mathrm{M} \mathrm{CaCl}_{2}, 30 \mu \mathrm{M} \mathrm{MnSO}_{4}, 10 \mu \mathrm{gl}^{-1}\right.$ chloramphenicol) and grown to exponential phase that equated to $1 \times 10^{8} \mathrm{CFU}$ $\mathrm{ml}^{-1}$. Each $10 \mathrm{ml}$ culture were then split into three, $2 \mathrm{ml}$ cultures ( $15 \mathrm{ml}$ tube) with either (i) no $\mathrm{ZnSO}_{4}$, (ii) $25 \mu \mathrm{M} \mathrm{ZnSO}$ or (iii) $25 \mu \mathrm{M} \mathrm{ZnSO}_{4}$ and $10 \mu \mathrm{M}$ TPEN was added. The cultures were reincubated and grown for $24 \mathrm{~h}$ before the fluorescence was determined as described above.

Antibiotic resistance profiling. For vancomycin gradient, assays were performed as described except a $0-2 \mu \mathrm{g} \mathrm{ml}^{-1}$ gradient was used ${ }^{51}$. After $24 \mathrm{~h}$ incubation at 
$37^{\circ} \mathrm{C}$, plates were imaged. With the exception of Vitek 2 and Etests, all antibiotic susceptibility testing of strains was performed in triplicate. To access the susceptibility to a range of antibiotics, strains were tested on a Vitek 2 Gram Positive ID card (AST-P612; Biomerieux) as per manufacturer's instructions. Extended glycopeptide susceptibilities were determined with vancomycin Etest strips (Biomerieux) using a 2.0 McFarland inoculum on thick BHI agar and incubation of $48 \mathrm{~h}$ at $37^{\circ} \mathrm{C}$.

Supernatant protein precipitation and sodium dodecyl sulfate-polyacrylamide gel electrophoresis (SDS-PAGE) analysis. Supernatant proteins from overnight culture or $\mathrm{OD}_{600}=0.8 \mathrm{LB}$ cultures were precipitated with $10 \%$ trichloroacetic acid at $4{ }^{\circ} \mathrm{C}$ for $1 \mathrm{~h}$. Pellets were washed with $1 \mathrm{ml}$ of ice-cold acetone and air dried at room temperature. Precipitated proteins were resuspended in $2 \times$ SDS-PAGE loading buffer, and prior to loading, the samples were equilibrated with an equal volume of $1 \mathrm{M}$ Tris.Cl [8.0]. Zymogram analysis of the supernatant proteins was conducted as described ${ }^{52}$.

Cloning, expression and purification of the GST-WalK PAS domain in E. coli. WalK-PAS FULL (residues Val251-Arg376) was PCR amplified from NRS384 genomic DNA with oligonucleotides WalK-PAS ${ }^{\mathrm{FULL}}-\mathrm{F} / \mathrm{WalK}-\mathrm{PAS}^{\mathrm{FULL}}-\mathrm{R}$ and cloned into pGEX-2T (BamHI/EcoRI) to yield a N-terminally tagged GST-PAS construct. For WalK-PAS ${ }^{\text {TRUNC }}$ and WalK-PAS ${ }^{\text {TRUNC H271Y }}$ (residues Asp266-Glu371), codon-optimised genes were ordered from GeneArt, and these were sub-cloned as BamHI/EcoRI fragments into pGEX-2T yielding pGEX-2T (WalK-PASTRUNC) and pGEX-2T(WalK-PAS TRUNC H271Y). Overnight cultures of BL21 containing the different GST constructs were diluted 1:100 into 21 of TB at $37^{\circ} \mathrm{C}$ at $180 \mathrm{rpm}$ and grown to $\mathrm{OD}_{600}$ of 0.8 . The culture was then induced with $0.4 \mathrm{mM}$ IPTG and shifted to $16^{\circ} \mathrm{C}$ at $120 \mathrm{rpm}$ for $10 \mathrm{~h}$ (final $\mathrm{OD}_{600}$ of $\sim 3$ ). The cells were harvested by centrifugation at $5000 \times g$ for $15 \mathrm{~min}$ at $4^{\circ} \mathrm{C}$. The pellet was resuspended in $50 \mathrm{ml}$ of GST lysis buffer (50 mM Tris.Cl [8.0], $500 \mathrm{mM} \mathrm{NaCl}$, $1 \mathrm{mM}$ EDTA) containing $1 \mathrm{mg} \mathrm{ml}^{-1}$ lysozyme plus $1 \mathrm{U} \mathrm{ml}^{-1}$ DNase A and incubated for $30 \mathrm{~min}$ on ice. Cells were lysed at $40 \mathrm{kpsi}$ in a cell disruptor (Constant Systems), then cell debris was removed by centrifugation at $39,000 \mathrm{~g}$ for $30 \mathrm{~min}$ at $4{ }^{\circ} \mathrm{C}$. The supernatant was collected and passed through GST-affinity resin in a gravity-flow column (Bio-Rad). The resin was then washed with two column volumes of GST lysis buffer followed by equilibration with 1 column volume of TCB (20 mM Tris [8.0], $500 \mathrm{mM} \mathrm{NaCl}, 1 \mathrm{mM} \mathrm{CaCl}_{2}$ ). WalK-PAS was eluted by oncolumn digestion with $20 \mathrm{ml}$ of TCB containing $200 \mathrm{U}$ of thrombin. Thrombin digestion was carried out by incubating the column at room temperature for $30 \mathrm{~min}$ followed by $30 \mathrm{~min}$ at $37^{\circ} \mathrm{C}$ and $60 \mathrm{~min}$ at room temperature. The liberated PAS domain was purified by size exclusion chromatography using a Superdex 75 column. Purified PAS domains were concentrated using an Amicon centrifugal concentrator with a $10-\mathrm{kDa}$ size cut-off. Protein purity was always $>95 \%$ as determined by SDS-PAGE with a yield of about $20 \mathrm{mg} / \mathrm{l}$.

\section{Expression and purification of selenomethionine-labelled WalK-PAS domain.} A single colony of BL21+pGEX-2T(WalK-PAS ${ }^{\mathrm{FULL}}$ ) was picked and used to inoculate $10 \mathrm{ml}$ of pre-warmed LB at $37^{\circ} \mathrm{C}$ for $6 \mathrm{~h}(180 \mathrm{rpm})$. Minimal media $(100 \mathrm{ml})$ was inoculated with $500 \mu \mathrm{l}$ of this pre-culture. Cells were grown overnight at $37^{\circ} \mathrm{C}$ with shaking at $180 \mathrm{rpm}$, then $25 \mathrm{ml}$ of culture was added to 21 minimal medium and incubated at $30^{\circ} \mathrm{C}$. After further growth to $\mathrm{OD}_{600} \sim 0.7$, amino acid stock solution containing selenomethionine was added, then cells were grown for $1 \mathrm{~h}$ before induction with $0.4 \mathrm{mM}$ IPTG. Cells were harvested by centrifugation at $6000 \times g$ for $20 \mathrm{~min}$ at $4{ }^{\circ} \mathrm{C}$ and the resulting cell pellet stored at $-80^{\circ} \mathrm{C}$. The SeMet-labelled WalK-PAS ${ }^{\mathrm{FULL}}$ was purified as described above. Labelling was confirmed by peptide mapping through matrix-assisted laser desorption/ionisation (MALDI) MS. A total of $10 \mu \mathrm{g}$ of purified protein was subjected to digestion for $16 \mathrm{~h}$ with $125 \mathrm{ng}$ porcine trypsin (MS-grade, Promega) in $200 \mathrm{mM}$ tetraetyl ammonium bicarbonate [8.0] containing $10 \%$ acetonitrile. The peptide digest was mixed in a $1: 1 \mathrm{v} / \mathrm{v}$ using solution of $\alpha$-cyano- 4 hydroxycinamic and matrix $\left(5 \mathrm{mg} \mathrm{ml}^{-1}\right.$ in $50 \%$ acetonitrile in $0.1 \%$ trifluoroacetic acid), spotted directly onto stainless steel MALDI target and MALDI-time of flight (TOF)/TOF spectra acquired using a Model 4700 Proteomic Analyser (Applied Biosystems). For the digested peptides, the mass spectrometer was operated in reflector positive ionisation mode using a $\mathrm{m} / \mathrm{z}$ range of 700-4000. The MS peak list was extracted in the GPS explorer software using the default parameters. A list of theoretical tryptic peptides obtained with the program GPMAW (allowing for one missed cleavage) was used to interpret the MS spectra based on an average increase in $\mathrm{m} / z$ of $47 \mathrm{Da}$ for each selenomethionine residue.

Cloning, expression and purification of the cytoplasmic domains of $\mathbf{H i s}_{6}$-WalK in E. coli. The cytoplasmic region of WalK (residues 208-608 ${ }^{\mathrm{TAA}}$ ), with or without the H271Y mutation, was PCR amplified from NRS384 genomic DNA with primers WalK208-F/WalK ${ }^{\mathrm{TAA}}-\mathrm{R}$ (WalKCYTO ) or WalK $208 \mathrm{~F} / \mathrm{IM} 8 / \mathrm{IM} 9 / \mathrm{WalK}^{\mathrm{TAA}}-\mathrm{R}$ (WalK ${ }^{\mathrm{CYTO}-\mathrm{H} 271 \mathrm{Y}}$ ), with the latter assembled by SOE-PCR. The products were ligated into pET19b after digestion with $\mathrm{NdeI}$ and BamHI and then transformed into DH5a. After transfer of the sequence-verified construct into BL21, an overnight culture in TB of pET19b(WalKCYTO) or pET19b(WalKCYTO-H271Y) was diluted 1:100 in 21 of TB. Cells were grown at $37^{\circ} \mathrm{C}$ and $180 \mathrm{rpm}$ until $\mathrm{OD}_{600} \approx 0.2$ and then the growth temperature was reduced to $16^{\circ} \mathrm{C}$. Protein production was induced with $0.2 \mathrm{mM}$ IPTG at an $\mathrm{OD}_{600} \approx 1$, then cultures were grown overnight at $16^{\circ} \mathrm{C}$. Cells were harvested by centrifugation at $8000 \times g$ for $8 \mathrm{~min}$ at $4{ }^{\circ} \mathrm{C}$, then stored at $-80^{\circ} \mathrm{C}$. Frozen cells were resuspended in native lysis buffer $(50 \mathrm{mM}$ Tris [7.5], $500 \mathrm{mM} \mathrm{NaCl}, 25 \mathrm{mM}$ imidazole, $5 \mathrm{mM}$ EDTA), passed through a cell disruptor (Constant Systems) at $35 \mathrm{kpsi}$ and then cell debris removed by centrifugation at $39,000 \times g$ for $30 \mathrm{~min}$ at $4{ }^{\circ} \mathrm{C}$. The supernatant was loaded on a NiNTA column equilibrated with equilibration buffer $(25 \mathrm{mM}$ Tris [7.5], $25 \mathrm{mM}$ imidazole, $500 \mathrm{mM} \mathrm{NaCl}$ ). The column was washed with equilibration buffer followed by a wash step with pre-elution buffer $(25 \mathrm{mM}$ Tris [7.5], $90 \mathrm{mM}$ imidazole, $500 \mathrm{mM} \mathrm{NaCl}, 5 \mathrm{mM}$ EDTA). WalK protein was then eluted in the same buffer containing $250 \mathrm{mM}$ imidazole and immediately desalted over a HiPrep $26 / 10$ desalting column into desalt buffer ( $25 \mathrm{mM}$ Tris [7.5], $150 \mathrm{mM} \mathrm{NaCl}, 2 \%$ glycerol).

Crystallisation of the WalK PAS domain. The purified WalK-PAS ${ }^{\mathrm{FULL}}$ was concentrated to $15 \mathrm{mg} \mathrm{ml}^{-1}$ and then screened for optimal crystallisation conditions. The protein was crystallised from conditions using the PACT screen (Molecular Dimensions). Plates were set up using a Mosquito crystallisation robot (TTP Labtech). Crystallisation conditions were then refined with the best crystals formed in PAS buffer (100 mM Tris.Cl [8.0], $20 \mathrm{mM} \mathrm{ZnCl}_{2}$ and $26 \%$ (w/v) PEG 3350) at $20^{\circ} \mathrm{C}$. Notably, the presence of $\mathrm{ZnCl}_{2}$ was essential for crystal formation. WalK-PAS ${ }^{\mathrm{FULL}}$ crystals were mounted using a $15-\mu \mathrm{M}$-nylon cryo-loop (Hampton Research) and soaked in cryoprotectant solutions consisting of the crystallisation PAS buffer containing 20\% PEG 400 for 10 min each and flash-cooled in liquid nitrogen and stored in liquid nitrogen for X-ray diffraction studies. The shorter WalK-PAS ${ }^{\text {TRUNC }}$ domain (residues 266-371) was crystallised using the same conditions.

Model building and refinement. The structure of the PAS domain was solved by single-wavelength anomalous dispersion (SAD) using SeMet-labelled WalKPAS FULL. A single crystal was used to collect a highly redundant data set at the peak wavelength for selenium at the Australian Synchrotron (Supplementary Table 1). Data were processed with MOSFLM ${ }^{53}$ and scaled with SCALA ${ }^{54}$. Heavy atom sites were found and refined to find the phase, and an initial model was built using the AutoSol program in PHENIX ${ }^{55}$. The resulting initial model was then subjected to multiple rounds of refinement in PHENIX and rebuilding in COOT $^{56}$ using a higher-resolution data set (WalK-PAS ${ }^{\mathrm{FULL}}$, Supplementary Table 1) collected with longer exposure times. The structure of the unlabelled shorter version of the WalK-PAS ${ }^{\text {TRUNC }}$ domain was subsequently determined by molecular replacement using PHASER ${ }^{53}$. Phasing and refinement statistics are given in Supplementary Table 1. In order to unequivocally identify the bound metal as $\mathrm{Zn}$, diffraction data were collected from an additional WalK-PAS ${ }^{\mathrm{FULL}}$ crystal on Australian Synchrotron beamline MX2 at two energies at the Zn $K$ edge (Supplementary Table 1, WalK-PAS ${ }^{\mathrm{FULL}} \mathrm{Zn}$ peak edge, WalK-PAS ${ }^{\mathrm{FULL}} \mathrm{Zn}$ low energy edge). The data were indexed and integrated with XDS and scaled with AIMLESS. Anomalous difference Fourier maps were calculated with these data with FFT $^{57-59}$.

Molecular dynamics. A membrane-bound homology model of the WalK complex (sequence based on Uniprot entry Q2G2U4) was constructed with VMD 60 and SwissModel (https://swissmodel.expasy.org) using PDB crystal structures 4MN6 (residues 266-320, identity 100\%), 5IS1 (residues 33-182, identity 100\%) and 4I5S (residues 223-599, identity 44\%) as templates ${ }^{61}$. Missing regions 1-33, 183-222 and 600-608 were independently modelled using secondary structure prediction program Psipred (http://bioinf.cs.ucl.ac.uk/psipred/) as a guide as no significant similar structures were available. The missing $\mathrm{N}$ terminal and middle sections were modelled as trans-membrane helices, while the missing C-terminus was included as disordered (Supplementary Fig. 4). Models were fully solvated, ionised with $0.15 \mathrm{M}$ $\mathrm{NaCl}$ and embedded through a phosphatidylcholine lipid bilayer. One version had $\mathrm{Zn}^{2+}$ ions bound to each of the cytoplasmic PAS domains, while the other was metal free. Initial dimensions were $127 \times 127 \times 260 \AA$ containing 385,994 and 385,992 atoms, respectively. Molecular simulations were performed using NAMD2.12 ${ }^{62}$ with the CHARM36 force field ${ }^{63}$

Simulations were run with periodic boundary conditions using the ensembles at $37^{\circ} \mathrm{C}$ and 1 bar pressure employing Langevin dynamics. Long-range Coulomb forces were computed with the Particle Mesh Ewald method with a grid spacing of $1 \AA$. Time steps of 2 fs were used with non-bonded interactions calculated every 2 fs and full electrostatics every $4 \mathrm{fs}$ while hydrogens were constrained using the SHAKE algorithm. The cut-off distance was $12 \AA$ with a switching distance of $10 \AA$ and a pair-list distance of $14 \AA$. Pressure was controlled to $1 \mathrm{~atm}$ using the Nosé-Hoover Langevin piston method employing a piston period of $100 \mathrm{fs}$ and piston decay of $50 \mathrm{fs}$. Trajectory frames were captured every $100 \mathrm{ps}$. The zinc-free model was simulated for $160 \mathrm{~ns}$ while the $\mathrm{Zn}^{2+}$-bound model was simulated for $200 \mathrm{~ns}$. Simulations were unconstrained apart from weak harmonic constraints holding the $\mathrm{Zn}^{2+}$ ions in the bound position in the zinc-containing model. Dihedral angles between the cytoplasmic PAS and catalytic domains were measured over the course of the simulations with VMD. 
In vitro $\mathbf{Z n}^{\mathbf{2}+}$-loading assays. Metal-loading assays were performed on purified apo-WalK-PASTRUNC and WalK-PAS ${ }^{T R U N C-H 271 Y}(30 \mu \mathrm{M})$ by mixing with tenfold molar excess $\mathrm{Zn}^{2+}\left(300 \mu \mathrm{M} \mathrm{ZnSO}_{4}\right)$ in a total volume of $2 \mathrm{ml}$ in $20 \mathrm{mM}$ MOPS [7.2] and $100 \mathrm{mM} \mathrm{NaCl}$ for $60 \mathrm{~min}$ at $4{ }^{\circ} \mathrm{C}$. The sample was desalted on a PD10 column (GE Healthcare) into the above buffer, and then the protein concentration was determined. Samples containing $10 \mu \mathrm{M}$ total protein were prepared in $3.5 \%$ $\mathrm{HNO}_{3}$ and boiled for $15 \mathrm{~min}$ at $95^{\circ} \mathrm{C}$. Samples were then cooled and centrifuged for $20 \mathrm{~min}$ at $14,000 \times g$. The supernatant was then analysed by ICP-MS (Agilent $8900 \mathrm{QQQ})$, and the protein:metal ratio was determined.

Cell volume and concentration determination. Scanning electron microscopy was used to determine the dimensions of the bacterial cell. Bacteria were grown, as described above, harvested and then prepared for and analysed by a Philips XL30 FEG scanning electron microscope as described ${ }^{64}$. Briefly, cell measurements were obtained using instrument software and used to calculate volume according to the method of Zhou and coworkers ${ }^{65}$, assuming a prolate spheroid shape:

$$
V=\frac{4}{3} \pi a b^{2}
$$

where $a$ and $b$ are the dimensions long and short axes of the cell, respectively. The total quotient of transition metal ions was then derived using cell volume ( $V$, litres), where the total cell density is known (CFU), and concentration of metal ions in a sample of known volume and known number of cells ( $M$, moles).

$$
C=\frac{(M / \mathrm{CFU})}{V}
$$

The derived concentration represents mean concentration of metal ion per cell of mean dimensions $\left(\mathrm{mmol} \mathrm{l}^{-1}\right)$.

Determination of $\boldsymbol{K}_{\mathrm{D}}$ for $\mathbf{Z n}^{\mathbf{2}+}$ with WalK-PAS ${ }^{\mathrm{FULL}}$. Excitation-emission spectra were determined on a FLUOStar Omega (BMG Labtech) at $301 \mathrm{~K}$ using black halfvolume 384-well microtitre plates (Greiner Bio One). All experiments were performed in $20 \mathrm{mM}$ MOPS (pH 6.7) and $50 \mathrm{mM} \mathrm{NaCl}$ with FluoZin-3 (Life Technologies) at a concentration of $150 \mathrm{nM}$. Deionised water and buffers were prepared and treated with Chelex-100 (Sigma-Aldrich) to avoid metal contamination. Filters used for FluoZin-3 were excitation $(485 \pm 10 \mathrm{~nm})$ and emission $(520 \pm 5 \mathrm{~nm})$. To determine the dissociation constant between a metal $(X)$ and FluoZin-3 $(F)$, we considered the following equilibria:

$$
F+X \stackrel{K_{\mathrm{D} . X}}{\longleftrightarrow} F . X
$$

where, for a metal that increases the fluorescence of the probe by $>\sim 10 \%$, the following equation, which is an exact analytical relationship derived from the mass action equation for the formation of a 1:1 complex between probe and metal, was used to estimate the dissociation constant, $K_{\mathrm{D} . \mathrm{X}}$

$$
\frac{f-f_{\min }}{f_{\max }-f}=\frac{[X]}{K_{\mathrm{D} . X}}
$$

where $f$ is the measured fluorescence intensity in the presence of metal, $f_{\max }$ the fluorescence in the presence of saturating metal and $f_{\min }$ the fluorescence in the absence of metal. In all cases, a low concentration $(150 \mathrm{nM})$ of probe was used and we assumed that the free metal concentration was equal to the added metal concentration. The mean \pm s.e.m. $(n=6) K_{\mathrm{D}}$ determined for FluoZin-3 with $\mathrm{ZnSO}_{4}$ in the buffer system employed in this study was determined to be $257 \pm 47 \mathrm{nM}$. Competition by WalK-PAS FULL for $\mathrm{Zn}^{2+}$ binding was assessed by monitoring the decrease in the fluorescence of $150 \mathrm{nM}$ FluoZin-3- $\mathrm{Zn}^{2+}$ in response to increasing apo-WalK-PAS $\mathrm{FULL}$ concentrations and analysed using $\log _{10}$ [inhibitor] versus response model, with the experimentally derived $K_{\mathrm{D}}$ for FluoZin- 3 with $\mathrm{Zn}^{2+}$, in Graphpad Prism to determine the $K_{\mathrm{D}}$ value for $\mathrm{Zn}^{2+}$ binding.

Autophosphorylation assay. WalK ${ }^{\mathrm{CYTO}}$ or $\mathrm{WalK}^{\mathrm{CYTO}-\mathrm{H} 271 \mathrm{Y}}(1 \mu \mathrm{g})$ were incubated at room temperature in $15 \mu$ phosphorylation buffer $(25 \mathrm{mM}$ Tris, $300 \mathrm{mM}$ $\mathrm{NaCl}, 1 \mathrm{mM}$ TCEP, $20 \mathrm{mM} \mathrm{KCl}, 10 \mathrm{mM} \mathrm{MgCl}_{2}, \mathrm{pH}$ 8). Phosphorylation reactions were started by adding $1 \mu \mathrm{l}$ of radiolabelled ATP mixture $\left(2.5 \mu \mathrm{Ci}\left[\gamma^{-32} \mathrm{P}\right]\right.$-ATP and $5 \mu \mathrm{M} \mathrm{ATP}$ ) to the protein sample, which was then incubated for $60 \mathrm{~min}$ at room temperature. Reactions were stopped by adding $5 \mu$ l of $3 \times$ SDS-loading buffer, then samples were analysed on a $12 \%$ SDS-PAGE gel, followed by autoradiography. The intensity of phosphorylated protein bands was determined using the Quantity One software (Bio-Rad)

Detection of WalR phosphorylation using Phos-tag SDS-PAGE and western blot. Overnight cultures of NRS384, WalK ${ }^{\mathrm{H} 271 \mathrm{Y}}, \Delta s t p 1$ or $\Delta p k n B$ containing either the empty vector pRAB11, pRAB11 walR ${ }^{\mathrm{FLAG}}$ or pRAB11 walR $\mathrm{D} 53 \mathrm{~A}-\mathrm{FLAG}$ were diluted 1:100 into $100 \mathrm{ml}$ of TSB containing $10 \mu \mathrm{g} \mathrm{ml}^{-1}$ chloramphenicol and 0.4 $\mu \mathrm{M}$ ATc. Cultures were then grown to the start of stationary phase $\left(\mathrm{OD}_{600} \sim 4.0\right)$. For the chromosomally tagged WalR ${ }^{\mathrm{FLAG}}$ strains, overnight TSB cultures were diluted to $\mathrm{OD}_{600}=0.01$ in 1 litre of TSB and samples were taken after 110 (early $\log ), 150(\mathrm{mid} \log ), 180(\mathrm{mid} \log ), 240$ (late $\log$ ) and $420 \mathrm{~min}$ (early stationary phase). Samples were mixed with one sample volume of ice-cold ethanol:acetone and harvested by centrifugation at $7300 \times g$ for $5 \mathrm{~min}$ at $4{ }^{\circ} \mathrm{C}$. The cells were washed with $20 \mathrm{ml}$ of milliQ water and resuspended in $500 \mu \mathrm{l}$ of TBS (50 mM Tris.Cl [7.5], $150 \mathrm{mM} \mathrm{NaCl}$ ). Cells were disrupted by bead beating three times at $5000 \mathrm{rpm}$ for $30 \mathrm{~s}$ (Precellys 24, Bertin Instruments) and then the lysates were centrifuged at $11,000 \times g$ for $5 \mathrm{~min}$ at $4{ }^{\circ} \mathrm{C}$. A total of $25 \mu \mathrm{g}$ of protein was loaded on an $8 \%$ SDSPAGE gel containing $50 \mu \mathrm{M}$ Phos-tag acrylamide (Wako Chemicals) and $100 \mu \mathrm{M}$ $\mathrm{MnCl}_{2}$. The gel was run according to the manufacturer's instructions (Wako Chemicals). To remove manganese ions after electrophoresis, the gel was washed two times for $15 \mathrm{~min}$ with transfer buffer (25 mM Tris [8.3], $192 \mathrm{mM}$ glycine, $20 \%$ methanol) containing $1 \mathrm{mM}$ EDTA and once with transfer buffer without EDTA. The separated proteins were blotted onto a PVDF membrane using the Trans-Blot ${ }^{\circledR}$ Turbo $^{\text {Dx }}$ transfer system (Bio-Rad) according to the manufacturer's instructions. The membrane was treated with blocking buffer (5\% EasyBlocker (GeneTex) in TBS, $0.05 \%$ Tween 20) for $16 \mathrm{~h}$ at $4{ }^{\circ} \mathrm{C}$ and then with blocking buffer containing 1:500 mouse anti-FLAG ${ }^{\oplus}$ M2-Peroxidase (HRP) monoclonal antibody (Sigma) for $1 \mathrm{~h}$ at room temperature. The membrane was washed three times with TBS containing $0.05 \%$ Tween 20 and bound antibody was detected using the WesternSure ${ }^{\circledast}$ PREMIUM Chemiluminescent Substrate and the C-DiGit ${ }^{\oplus}$ Blot Scanner (LI-COR Biotechnology). The ratio of phosphorylated WalR was calculated by quantification of the western blot bands using GelAnalyser 2010a.

Reporting summary. Further information on research design is available in the Nature Research Reporting Summary linked to this article.

\section{Data availability}

All sequencing data used in this study have been deposited in the National Center for Biotechnology Information BioProject database under accession code PRJNA486581. Atomic coordinates and data for the cytoplasmic PAS domain of WalK have been deposited in the Protein Data Bank under accession numbers 4MN5 (WalK-PASFULL; residues 251-376) and 4MN6 (WalK-PASTRUNC; residues 266-371).

\section{Code availability}

The software used for mutation detection is available here: https:/github.com/tseemann/ snippy.

Received: 30 August 2018 Accepted: 5 June 2019

Published online: 11 July 2019

\section{References}

1. Tong, S. Y., Davis, J. S., Eichenberger, E., Holland, T. L. \& Fowler, V. G. Jr. Staphylococcus aureus infections: epidemiology, pathophysiology, clinical manifestations, and management. Clin. Microbiol. Rev. 28, 603-661 (2015).

2. Howden, B. P., Davies, J. K., Johnson, P. D., Stinear, T. P. \& Grayson, M. L. Reduced vancomycin susceptibility in Staphylococcus aureus, including vancomycin-intermediate and heterogeneous vancomycin-intermediate strains: resistance mechanisms, laboratory detection, and clinical implications. Clin. Microbiol. Rev. 23, 99-139 (2010).

3. Kelley, P. G., Gao, W., Ward, P. B. \& Howden, B. P. Daptomycin nonsusceptibility in vancomycin-intermediate Staphylococcus aureus (VISA) and heterogeneous-VISA (hVISA): implications for therapy after vancomycin treatment failure. J. Antimicrob. Chemother. 66, 1057-1060 (2011).

4. Chua, K. \& Howden, B. P. Treating Gram-positive infections: vancomycin update and the whys, wherefores and evidence base for continuous infusion of anti-Gram-positive antibiotics. Curr. Infect. Dis. Rep. 22, 525-534 (2009).

5. Holmes, N. E. et al. Antibiotic choice may not explain poorer outcomes in patients with Staphylococcus aureus bacteremia and high vancomycin minimum inhibitory concentrations. J. Infect. Dis. 204, 340-347 (2011).

6. Dubrac, S., Bisicchia, P., Devine, K. M. \& Msadek, T. A matter of life and death: cell wall homeostasis and the WalKR (YycGF) essential signal transduction pathway. Mol. Microbiol. 70, 1307-1322 (2008).

7. Bem, A. E. et al. Bacterial histidine kinases as novel antibacterial drug targets. ACS Chem. Biol. 10, 213-224 (2015).

8. Fabret, C. \& Hoch, J. A. A two-component signal transduction system essential for growth of Bacillus subtilis: implications for anti-infective therapy. J. Bacteriol. 180, 6375-6383 (1998).

9. Martin, P. K., Li, T., Sun, D., Biek, D. P. \& Schmid, M. B. Role in cell permeability of an essential two-component system in Staphylococcus aureus. J. Bacteriol. 181, 3666-3673 (1999).

10. Dubrac, S. \& Msadek, T. Identification of genes controlled by the essential YycG/YycF two-component system of Staphylococcus aureus. J. Bacteriol. 186, 1175-1181 (2004)

11. Howell, A. et al. Genes controlled by the essential YycG/YycF two-component system of Bacillus subtilis revealed through a novel hybrid regulator approach. Mol. Microbiol. 49, 1639-1655 (2003). 
12. Hancock, L. E. \& Perego, M. Systematic inactivation and phenotypic characterization of two-component signal transduction systems of Enterococcus faecalis V583. J. Bacteriol. 186, 7951-7958 (2004).

13. Kallipolitis, B. H. \& Ingmer, H. Listeria monocytogenes response regulators important for stress tolerance and pathogenesis. FEMS Microbiol Lett. 204, 111-115 (2001).

14. Villanueva, M. et al. Sensory deprivation in Staphylococcus aureus. Nat. Commun. 9, 523 (2018).

15. Fukuchi, K. et al. The essential two-component regulatory system encoded by $y y c F$ and $y y c G$ modulates expression of the ftsAZ operon in Bacillus subtilis. Microbiology 146(Pt 7), 1573-1583 (2000).

16. Dubrac, S., Boneca, I. G., Poupel, O. \& Msadek, T. New insights into the WalK/WalR (YycG/YycF) essential signal transduction pathway reveal a major role in controlling cell wall metabolism and biofilm formation in Staphylococcus aureus. J. Bacteriol. 189, 8257-8269 (2007).

17. Howden, B. P. et al. Genomic analysis reveals a point mutation in the twocomponent sensor gene graS that leads to intermediate vancomycin resistance in clinical Staphylococcus aureus. Antimicrob. Agents Chemother. 52, 3755-3762 (2008).

18. Mwangi, M. M. et al. Tracking the in vivo evolution of multidrug resistance in Staphylococcus aureus by whole-genome sequencing. Proc. Natl Acad. Sci. USA 104, 9451-9456 (2007).

19. Neoh, H. M. et al. Mutated response regulator graR is responsible for phenotypic conversion of Staphylococcus aureus from heterogeneous vancomycin-intermediate resistance to vancomycin-intermediate resistance. Antimicrob. Agents Chemother. 52, 45-53 (2008).

20. Hu, Q., Peng, H. \& Rao, X. Molecular events for promotion of vancomycin resistance in vancomycin intermediate Staphylococcus aureus. Front. Microbiol. 7, 1601 (2016).

21. Shoji, M. et al. walK and $c l p P$ mutations confer reduced vancomycin susceptibility in Staphylococcus aureus. Antimicrob. Agents Chemother. 55, 3870-3881 (2011).

22. Howden, B. P. et al. Evolution of multidrug resistance during Staphylococcus aureus infection involves mutation of the essential two component regulator WalKR. PLoS Pathog. 7, e1002359 (2011).

23. Hu, J. F., Zhang, X., Liu, X. Y., Chen, C. \& Sun, B. L. Mechanism of reduced vancomycin susceptibility conferred by walK mutation in communityacquired methicillin-resistant Staphylococcus aureus strain MW2. Antimicrob. Agents Chemother. 59, 1352-1355 (2015).

24. Fukushima, T. et al. A role for the essential YycG sensor histidine kinase in sensing cell division. Mol. Microbiol. 79, 503-522 (2011).

25. Bisicchia, P. et al. The essential YycFG two-component system controls cell wall metabolism in Bacillus subtilis. Mol. Microbiol. 65, 180-200 (2007). d.

26. Delaune, A. et al. The WalKR system controls major staphylococcal virulence genes and is involved in triggering the host inflammatory response. Infect. Immun. 80, 3438-3453 (2012).

27. Delaune, A. et al. Peptidoglycan crosslinking relaxation plays an important role in Staphylococcus aureus WalKR-dependent cell viability. PLoS ONE 6 , e17054 (2011).

28. Cameron, D. R., Jiang, J. H., Kostoulias, X., Foxwell, D. J. \& Peleg, A. Y. Vancomycin susceptibility in methicillin-resistant Staphylococcus aureus is mediated by YycHI activation of the WalRK essential two-component regulatory system. Sci. Rep. https://doi.org/10.1038/srep30823 (2016).

29. Szurmant, H., Mohan, M. A., Imus, P. M. \& Hoch, J. A. YycH and YycI interact to regulate the essential YycFG two-component system in Bacillus subtilis. J. Bacteriol. 189, 3280-3289 (2007).

30. Henry, J. T. \& Crosson, S. Ligand-binding PAS domains in a genomic, cellular, and structural context. Annu. Rev. Microbiol. 65, 261-286 (2011).

31. Poupel, O. et al. Transcriptional analysis and subcellular protein localization reveal specific features of the essential WalKR system in Staphylococcus aureus. PLoS ONE 11, e0151449 (2016).

32. Monk, I. R., Howden, B. P., Seemann, T. \& Stinear, T. P. Correspondence: Spontaneous secondary mutations confound analysis of the essential twocomponent system WalKR in Staphylococcus aureus. Nat. Commun. https:// doi.org/10.1038/ncomms14403 (2017).

33. Pasztor, L. et al. Staphylococcal major autolysin (Atl) is involved in excretion of cytoplasmic proteins. J. Biol. Chem. 285, 36794-36803 (2010).

34. Bose, J. L., Lehman, M. K., Fey, P. D. \& Bayles, K. W. Contribution of the Staphylococcus aureus Atl AM and GL murein hydrolase activities in cell division, autolysis, and biofilm formation. PLoS ONE https://doi.org/10.1371/ journal.pone.0042244 (2012).

35. Gong, W. M. et al. Structure of a biological oxygen sensor: A new mechanism for heme-driven signal transduction. Proc. Natl Acad. Sci. USA 95, 15177-15182 (1998).

36. Laitaoja, M., Valjakka, J. \& Janis, J. Zinc coordination spheres in protein structures. Inorg. Chem. 52, 10983-10991 (2013).
37. Hardt, P. et al. The cell wall precursor lipid II acts as a molecular signal for the Ser/Thr kinase PknB of Staphylococcus aureus. Int. J. Med. Microbiol. 307, 1-10 (2017).

38. Libby, E. A., Goss, L. A. \& Dworkin, J. The eukaryotic-like Ser/Thr kinase PrkC regulates the essential WalRK two-component system in Bacillus subtilis. PLoS Genet. https://doi.org/10.1371/journal.pgen.1005275 (2015).

39. Wang, C. et al. Mechanistic insights revealed by the crystal structure of a histidine kinase with signal transducer and sensor domains. PLoS Biol. https:// doi.org/10.1371/journal.pbio.1001493 (2013).

40. Jacob-Dubuisson, F., Mechaly, A., Betton, J. M. \& Antoine, R. Structural insights into the signalling mechanisms of two-component systems. Nat. Rev. Microbiol. https://doi.org/10.1038/s41579-018-0055-7 (2018).

41. Mullner, M. et al. A PAS domain with an oxygen labile $[4 \mathrm{Fe}-4 \mathrm{~S}](2+)$ cluster in the oxygen sensor kinase NreB of Staphylococcus carnosus. Biochemistry 47, 13921-13932 (2008).

42. Little, R., Martinez-Argudo, I. \& Dixon, R. Role of the central region of NifL in conformational switches that regulate nitrogen fixation. Biochem. Soc. Trans. 34, 162-164 (2006).

43. Cheung, J., Bingman, C. A., Reyngold, M., Hendrickson, W. A. \& Waldburger, C. D. Crystal structure of a functional dimer of the PhoQ sensor domain. J. Biol. Chem. 283, 13762-13770 (2008).

44. Grundling, A., Missiakas, D. M. \& Schneewind, O. Staphylococcus aureus mutants with increased lysostaphin resistance. J. Bacteriol. 188, 6286-6297 (2006).

45. Poupel, O., Proux, C., Jagla, B., Msadek, T. \& Dubrac, S. SpdC, a novel virulence factor, controls histidine kinase activity in Staphylococcus aureus. PLoS Pathog. 14, e1006917 (2018).

46. Echenique, J. R. \& Trombe, M. C. Competence repression under oxygen limitation through the two-component MicAB signal-transducing system in Streptococcus pneumoniae and involvement of the PAS domain of MicB. J. Bacteriol. 183, 4599-4608 (2001).

47. Zhang, Y., Werling, U. \& Edelmann, W. SLiCE: a novel bacterial cell extractbased DNA cloning method. Nucleic Acids Res. 40, e55 (2012).

48. Monk, I. R., Tree, J. J., Howden, B. P., Stinear, T. P. \& Foster, T. J. Complete bypass of restriction systems for major Staphylococcus aureus lineages. Mbio https://doi.org/10.1128/mBio.00308-15 (2015).

49. Monk, I. R., Shah, I. M., Xu, M., Tan, M. W. \& Foster, T. J. Transforming the untransformable: application of direct transformation to manipulate genetically Staphylococcus aureus and Staphylococcus epidermidis. mBio https://doi.org/10.1128/mBio.00277-11 (2012).

50. Bose, J. L., Fey, P. D. \& Bayles, K. W. Genetic tools to enhance the study of gene function and regulation in Staphylococcus aureus. Appl. Environ. Microbiol. 79, 2218-2224 (2013).

51. Lee, J. Y. H. et al. Global spread of three multidrug-resistant lineages of Staphylococcus epidermidis. Nat. Microbiol. https://doi.org/10.1038/s41564018-0230-7 (2018).

52. Monk, I. R., Cook, G. M., Monk, B. C. \& Bremer, P. J. Morphotypic conversion in Listeria monocytogenes biofilm formation: biological significance of rough colony isolates. Appl. Environ. Microbiol. 70, 6686-6694 (2004).

53. McCoy, A. J. et al. Phaser crystallographic software. J. Appl. Crystallogr. 40, 658-674 (2007).

54. Evans, P. R. An introduction to data reduction: space-group determination, scaling and intensity statistics. Acta Crystallogr. D Biol. Crystallogr. 67, 282-292 (2011).

55. Adams, P. D. et al. PHENIX: a comprehensive Python-based system for macromolecular structure solution. Acta Crystallogr. D Biol. Crystallogr. 66, 213-221 (2010).

56. Emsley, P., Lohkamp, B., Scott, W. G. \& Cowtan, K. Features and development of Coot. Acta Crystallogr. D Biol. Crystallogr. 66, 486-501 (2010).

57. Evans, P. R. \& Murshudov, G. N. How good are my data and what is the resolution? Acta Crystallogr. D Biol. Crystallogr. 69, 1204-1214 (2013).

58. Kabsch, W. XDS. Acta Crystallogr. D Biol Crystallogr. 66, 125-132, (2010).

59. Winn, M. D. et al. Overview of the CCP4 suite and current developments. Acta Crystallogr. D Biol. Crystallogr. 67, 235-242 (2011).

60. Humphrey, W., Dalke, A. \& Schulten, K. VMD: visual molecular dynamics. J. Mol. Graph. 14, 33-38 (1996). 27-38.

61. Kim, T. et al. Structural studies on the extracellular domain of sensor histidine kinase YycG from Staphylococcus aureus and its functional implications. J. Mol. Biol. 428, 3074-3089 (2016).

62. Phillips, J. C. et al. Scalable molecular dynamics with NAMD. J. Comput. Chem. 26, 1781-1802 (2005).

63. Brooks, B. R. et al. CHARMM: the biomolecular simulation program. J. Comput. Chem. 30, 1545-1614 (2009).

64. Begg, S. L. et al. Dysregulation of transition metal ion homeostasis is the molecular basis for cadmium toxicity in Streptococcus pneumoniae. Nat. Commun. 6, 6418 (2015). 
65. Zhou, X. et al. Bacterial division. Mechanical crack propagation drives millisecond daughter cell separation in Staphylococcus aureus. Science 348, 574-578 (2015).

66. Stivala, A., Wybrow, M., Wirth, A., Whisstock, J. C. \& Stuckey, P. J. Automatic generation of protein structure cartoons with Pro-origami. Bioinformatics 27, 3315-3316 (2011).

67. Baker, N. A., Sept, D., Joseph, S., Holst, M. J. \& McCammon, J. A. Electrostatics of nanosystems: application to microtubules and the ribosome. Proc. Natl Acad. Sci. USA 98, 10037-10041 (2001).

\section{Acknowledgements}

We acknowledge funding from the Australian National Health and Medical Research Council (Project Grant GNT1010776 and Principal Research Fellowship GNT1044414 to G.F.K.; Early Career Research Fellowship GNT1142695 to S.L.B., Senior Research Fellowship GNT1136021 to B.M.C.; Project Grants GNT1049192, GNT1129589 and GNT1145075 and Senior Research Fellowship GNT1105525 to T.P.S.; and Practioner Research Fellowship GNT1105905 to B.P.H.) and the Australian Research Council (Discovery Project DP170102102 and Future Fellowship FT170100006 to C.A.M.). Aspects of this research were undertaken on the Macromolecular Crystallography beamline MX2 at the Australian Synchrotron (Victoria, Australia) and we thank the beamline staff for their enthusiastic and professional support.

\section{Author contributions}

I.R.M., B.P.H., C.A.M., G.F.K. and T.P.S. designed the research. I.R.M. performed all experiments with S. aureus. S.L.B., J.R.M., L.K.R.S., S.J.P. and C.A.M. performed cloning experiments and protein function assays; J.Y.H.L. performed antibiotic sensitivity screens; M.G. and G.B. performed Phostag experiments; N.S., B.W., R.H., B.M.C., S.R.U., N.P., M.J.M. and G.F.K. performed protein structural analyses. M.K. performed molecular dynamics; T.S. performed bioinformatics analysis; I.R.M, N.S., S.L.B., C.A.M. and T. P.S. co-wrote the manuscript.

\section{Additional information}

Supplementary Information accompanies this paper at https://doi.org/10.1038/s41467019-10932-4.

Competing interests: The authors declare no competing interests.

Reprints and permission information is available online at http://npg.nature.com/ reprintsandpermissions/

Peer review information: Nature Communications thanks Toshihide Okajima, Victor Torres and other anonymous reviewer(s) for their contribution to the peer review of this work. Peer reviewer reports are available.

Publisher's note: Springer Nature remains neutral with regard to jurisdictional claims in published maps and institutional affiliations.

(c) (i) Open Access This article is licensed under a Creative Commons Attribution 4.0 International License, which permits use, sharing, adaptation, distribution and reproduction in any medium or format, as long as you give appropriate credit to the original author(s) and the source, provide a link to the Creative Commons license, and indicate if changes were made. The images or other third party material in this article are included in the article's Creative Commons license, unless indicated otherwise in a credit line to the material. If material is not included in the article's Creative Commons license and your intended use is not permitted by statutory regulation or exceeds the permitted use, you will need to obtain permission directly from the copyright holder. To view a copy of this license, visit http://creativecommons.org/ licenses/by/4.0/.

(C) The Author(s) 2019 